This article was published:

ChemistrySelect 2017, 2, 2565 - 2568

http://onlinelibrary.wiley.com/doi/10.100

2/slct.201700432/abstract

WILEY-VCH

\title{
Microwave-Mediated Sulfonium Ylide Cyclopropanation. Stereoselective Synthesis of Cyclopropa[c]pentalenes
}

\author{
Alberto López-Rodríguez, Gema Domínguez, Javier Pérez-Castells*
}

\begin{abstract}
Stereoselective sulfonium ylide cyclopropanation is a useful transformation due to the presence of this moiety in numerous bioactive compounds. The procedures described to date involve long reaction times and variable stereoselectivity outcomes. We show here that performing these reactions under MW mediation give excellent yields and selectivities after short reaction periods. The methodology is applied to different substrates including sterically hindered cyclopentenones, open chain unsaturated esters and pentalenones (Pauson-Khand products). From these latter substrates, highly connected cyclopropa[c]pentalenes were synthesized in good yields, including a precursor of natural product pentalenene.
\end{abstract}

\section{Introduction}

Cyclopropanes are found as a basic structural motif in naturally occurring compounds and in biologically active molecules. In addition, they can undergo many chemical transformations to give useful intermediates. The ylide cyclopropanation is one of the earliest developed and extensively studied synthetic procedures to build these structures. In particular sulfur ylides are important reagents useful for the preparation of cyclopropanes and epoxydes. ${ }^{1]}$ Chiral sulfur ylides have been used for the asymmetric version of this reaction. ${ }^{[2]}$ The experimental procedures reported to date generally consist of the treatment of a sulfonium salt with a base (generally DBU) to form the ylide, followed by prolonged reaction times to produce the cycloaddition reaction. The mechanistic course for the cyclopropanation, starts with the initial attack of the sulfur ylide to the electrophilic center of the Michael acceptor forming a betaine which gives the final cyclopropane after an intramolecular nucleophilic displacement (Scheme 1).

Previously reported mechanistic studies show that in this stepwise reaction the formation of the first $\mathrm{C}-\mathrm{C}$ bond is the rate determining step. ${ }^{[3]}$ The stereoselectivity of the reaction is determined, however, in the second step. Following studies reported by Aggarwal's group, two intermediate betaines $\mathbf{A}$ and B would be formed in a 1:1 ratio after the nucleophilic addition. The cyclization of betaine $\mathbf{B}$ is slow whereas A cyclizes faster. Due to the presence of a base, an epimerization of $\mathbf{B}$ is possible to give $\mathbf{A}$ leading generally to high diastereoselectivities favoring the exo isomer. ${ }^{[4]}$ This base-mediated epimerization is the

Facultad de Farmacia, Dpto. Química y Bioquímica, Universidad San Pablo CEU, Urb. Montepríncipe, Boadilla del Monte, 28668 Madrid. E-mail: ipercas@ceu.es

Supporting information for this article is given via a link at the end of the document responsible for a loss of enantioselectivity when using chiral sulfonium ylides, as the epimerization at the ester stereocenter ultimately leads to the opposite exo-enantiomer of the cyclopropane..$^{[5]}$

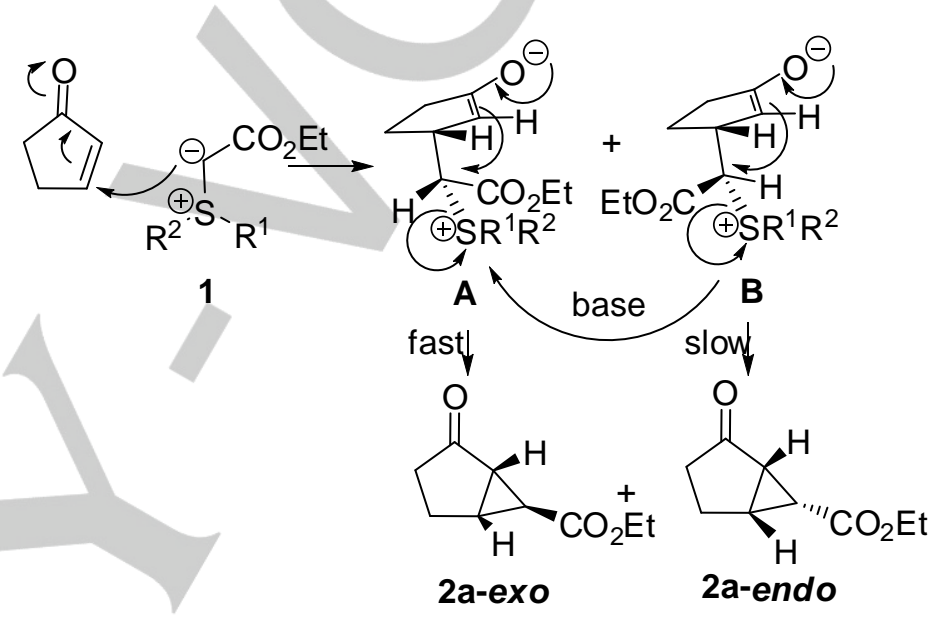

Scheme 1. Mechanism of ylide cyclopropanation.

The general long reaction conditions are an important drawback and conventional heating is scarcely used as it may cause undesired side reactions. A convenient alternative to circumvent this problem would be the conduction of the cyclopropanation in a microwave (MW) reactor ${ }^{[6]}$ Advantages compared to the conventional heating would be the rapid access to high reaction temperatures and fast cooling at the end of the reaction, improved purity profiles, and practical issues as the accurate control of reaction parameters.

Herein we show the feasibility of performing the sulfonium ylide cyclopropanation reaction under MW mediation which proceeds with excellent yields and selectivities after short reaction periods. We show the application of this methodology to the synthesis of highly connected tricycles including a precursor of natural product pentalenene.

\section{Results and Discussion}

used 2-cyclopentenone as a model substrate in order to optimize the ratio of sulfonium salt, base, temperature and time (Table 1). This reaction has been previously reported using reaction times from $8 \mathrm{~h}$ to more than $18 \mathrm{~h}$ at $\mathrm{rt}$ or $30^{\circ} \mathrm{C}$ giving yields going from $66-77 \% .^{[5,7]}$ It was described under conventional heating at $100{ }^{\circ} \mathrm{C}$ for $17 \mathrm{~h}$ giving $68 \%$ yield. ${ }^{[8]}$ We monitorized by NMR the conversion into final product. Final yield 
in pure product is shown. Among the different solvents used, (entries 1-4), only in DCM and chloroform did the reaction take place, giving the latter a better conversion and yield. Increasing the amount of salt and base (entry 5) only produced a marginal increment in conversion but lower yield. Raising the temperature allowed nearly or total conversions in 10 min achieving the best yields at $70^{\circ} \mathrm{C}$ (entry 6). These latter conditions were used with sulfonium salt $1 \mathrm{~b}$ but the yield achieved was lower (entry 7 ). At higher temperatures, a possible decomposition of starting materials was deleterious for the efficiency of this process (entries 8-9). Reduction of the reaction time to 1 minute did not give good results (entry 10 ). The reactions carried out during 60 minutes did not improve the results of those performed in 10 minutes (entries 11-12). Thus, selected conditions were: 1.1 equiv of salt, 1.1 equiv of $\mathrm{DBU}, 70^{\circ} \mathrm{C}, 10$ min (entry 6 ).<smiles>CCOC(=O)C1CC2CCC(=O)C21</smiles>

2a $(12: 1)$

2a-exo: $74 \%$

2a-endo: $6 \%$<smiles>CCOC1C2C(=O)CCC12C</smiles>

2d-endo (21\%)

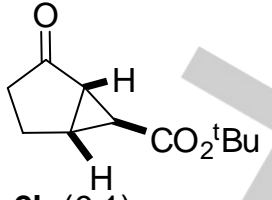

2b $(9: 1)$

2b-exo: $76 \%$ 2b-endo: $9 \%$

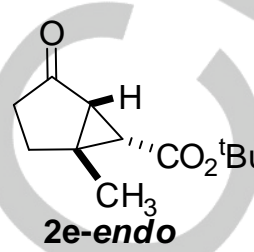

(22\%)<smiles>CCOC(=O)CC12C(=O)CCC1(C)C2(C)C(=O)OCC</smiles>

2c-endo: $16 \%$<smiles>COc1ccc(C(=O)C2CC3(CO)CCCC23)cc1</smiles>

Table 1. Optimization of reaction conditions. Synthesis of $\mathbf{2 a}$. $^{[a]}$

\begin{tabular}{|c|c|c|c|c|c|}
\hline No. & Solvent & $\begin{array}{c}\text { Temp. } \\
\left({ }^{\circ} \mathrm{C}\right)\end{array}$ & $\begin{array}{c}\text { Reaction } \\
\text { time } \\
\text { (min. })^{[b]}\end{array}$ & $\begin{array}{c}\text { Conversion } \\
(\%) \\
\text { (exo:endo) }^{[\mathrm{c}]}\end{array}$ & $\begin{array}{l}\text { Yield[d] } \\
2 a(\%)\end{array}$ \\
\hline 1 & DCM & 40 & 10 & $60(18: 1)$ & 23 \\
\hline 2 & $\mathrm{CHCl}_{3}$ & 40 & 10 & $80(15: 1)$ & 60 \\
\hline 3 & Toluene & 40 & 10 & n.d. & n.d. \\
\hline 4 & $\mathrm{H}_{2} \mathrm{O} / \mathrm{DMSO}$ & 40 & 10 & n.d. & n.d. \\
\hline 5 & $\mathrm{CHCl}_{3}^{[\mathrm{e}]}$ & 40 & 10 & $83(15: 1)$ & 58 \\
\hline 6 & $\mathrm{CHCl}_{3}$ & 70 & 10 & 95 (12:1) & 80 \\
\hline 7 & $\mathrm{CHCl}_{3}^{[f]}$ & 70 & 10 & $93(10: 1)$ & 69 \\
\hline 8 & $\mathrm{CHCl}_{3}$ & 100 & 10 & $100(8: 1)$ & 22 \\
\hline 9 & $\mathrm{CHCl}_{3}$ & 150 & 10 & n.d. & n.d. \\
\hline 10 & $\mathrm{CHCl}_{3}$ & 70 & 1 & $55(11: 1)$ & 40 \\
\hline 11 & $\mathrm{CHCl}_{3}$ & 70 & 60 & $95(12: 1)$ & 81 \\
\hline 12 & $\mathrm{CHCl}_{3}$ & 40 & 60 & $90(15: 1)$ & 60 \\
\hline
\end{tabular}

[a] Conditions: $\mathrm{DBU}$ (1.1 equiv), salt $1 \mathbf{a}\left(\mathrm{EtO}_{2} \mathrm{CCH}_{2}-\mathrm{S}^{+}\left(\mathrm{CH}_{3}\right)_{2}, \mathrm{Br} ; 1.1\right.$ equiv). [b] Ylide formation 30 min rt. [c] Ratio calculated from ${ }^{1} \mathrm{H}-\mathrm{NMR}$ spectra of the reaction crude. [d] Combined yield in pure exo and endo products. [e] DBU (2 equiv), 1a (2 equiv). [f] Salt $\mathbf{1 b}$ was used:

$$
\mathrm{EtO}_{2} \mathrm{C} \sim{ }_{\oplus}^{\mathrm{S}}>_{\Theta}^{\mathrm{Br}}
$$

With the optimized conditions in hand we extended the reaction to other substrates isolating products $\mathbf{2 b - f}$ and $\mathbf{4}$ (Figure 1 ). Good yields and exo-selectivities were achieved with products 2a-c. On the other hand $\mathbf{2 d - e}$ were isolated in poor yields and interestingly only as the endo isomers. The nucleophilic attack to the 3-methyl substituted cyclopentenone is sterically disfavoured which explains the low yield. In addition, the presence of the methyl group favors an intermediate of type B (Scheme 1), leading to the endo isomer. However this cyclopropanation reaction on 3-substituted unsaturated ketones is unprecedent to the best of our knowledge. Product $\mathbf{2 f}$ was isolated upon reaction with a benzoyl-stabilized sulfonium ylide in excellent yield, only detecting the exo isomer in the reaction mixture.

Figure 1. Cyclopropanation of 2-cyclopentenones.

In order to show the possibility of using chiral sulfonium ylides derived from camphor auxiliaries, we submitted 2cyclopentenone to a reaction with the ylide derived from 3 (Scheme 2a). ${ }^{\left[{ }^{9]}\right.}$ This reaction gave a crude mixture with a (19:1, exo:endo) ratio in product $\mathbf{2 b}$. The exo isomer was isolated with a $63 \%$ yield and with a moderate ee (32\%). ${ }^{[10]}$ In addition we prepared product $\mathbf{4}$ with excellent yield as a mixture of cis:trans isomers from an open chain substrate (Scheme $2 b$ ). (a)<smiles>O=C1C=CCC1</smiles><smiles>C[S+](CC(=O)OC(C)(C)C)C1C(O)C2(C)CCCC1(C)C2(C)C</smiles><smiles>CC(C)(C)OC(=O)C1C2CCC(=O)C21</smiles>

2b $(19: 1)$

(1S,5R,6S)-2b-exo: 63\% ee: $32 \%$

(b)

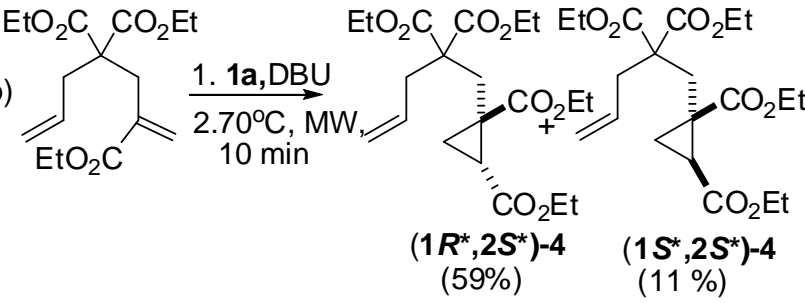

Scheme 2. Asymmetric synthesis of $\mathbf{2 b}$ and synthesis of $\mathbf{4}$.

We were interested in using this methodology for the synthesis of cyclopropa[c]pentalene systems as they are interesting highly compact structurally complex architectures. They can be used as synthetic intermediates for the synthesis of triquinane natural products. These are classic targets for total synthesis because 
of their biological activity. ${ }^{[11]}$ Thus, we prepared substrates $\mathbf{5 a - c}$, according to literature procedures, using the Pauson-Khand reaction. ${ }^{[12]}$ The reaction of the substrates with ylides from salts 1a and $\mathbf{1 c}$ is summarized in Scheme 3. Two out of the four possible diastereoisomers were formed in the three reactions, corresponding to the exo and endo cyclopropanes as shown by the coupling constants between the cyclopropanic protons $\mathrm{H} 1$ and $\mathrm{H} 1 \mathrm{a}$ ( $\mathrm{H} 5 \mathrm{a}$ and $\mathrm{H} 6$ for 6e-f). This means a total diastereoselective initial nucleophilic attack. The relative stereochemistry was established through NOE cross peaks. Main increments are shown for isomers $6 \mathbf{a}$. The reactions proceeded with good global yields despite the highly compact structures obtained and the formation of a quaternary carbon in the reaction. A reaction under conventional heating was performed with $5 \mathbf{a}$ (70 $\mathrm{C}$ heating for $18 \mathrm{~h})$ reaching a conversion of $45 \%$ and a total yield of $32 \%$ as a $1: 1$ mixture of isomers $6 \mathbf{a}$. Compounds $6 \mathbf{a}$ have been used as intermediates in the total synthesis of pentalenene. ${ }^{[13]}$

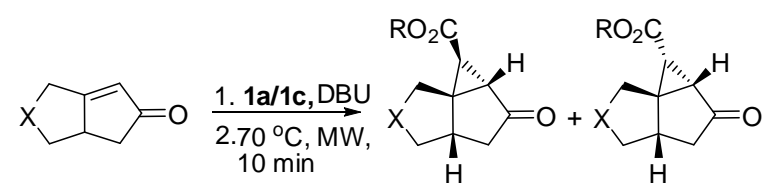

\begin{tabular}{|c|c|c|c|c|}
\hline & Yield: & exo & endo & ratio $^{[a]}$ \\
\hline \multirow{7}{*}{$\begin{array}{l}\text { 5a: } \mathrm{X}=\mathrm{C}\left(\mathrm{CH}_{3}\right)_{2} \\
\text { 5b: } \mathrm{X}=\mathrm{C}\left(\mathrm{CO}_{2} \mathrm{Et}\right)_{2} \\
\text { 5c: } \mathrm{X}=\mathrm{NTs}\end{array}$} & 6a: $\mathrm{R}=\mathrm{Et}, \mathrm{X}=\mathrm{C}\left(\mathrm{CH}_{3}\right)_{2}$ & $16 \%$ & $16 \%$ & $\overline{(1: 1)^{[b}}$ \\
\hline & 6a: $\mathrm{R}=\mathrm{Et}, \mathrm{X}=\mathrm{C}\left(\mathrm{CH}_{3}\right)_{2}$ & $31 \%$ & $29 \%$ & (1:1) \\
\hline & 6b: $\mathrm{R}={ }^{\mathrm{t}} \mathrm{Bu}, \mathrm{X}=\mathrm{C}\left(\mathrm{CH}_{3}\right)_{2}$ & $29 \%$ & $16 \%$ & $(3: 2)$ \\
\hline & 6c: $\mathrm{R}=\mathrm{Et}, \mathrm{X}=\mathrm{C}\left(\mathrm{CO}_{2} \mathrm{Et}\right)_{2}$ & $42 \%$ & $33 \%$ & $(5: 2)$ \\
\hline & 6d: $\mathrm{R}={ }^{\mathrm{t}} \mathrm{Bu}, \mathrm{X}=\mathrm{C}\left(\mathrm{CO}_{2} \mathrm{Et}\right)_{2}$ & $38 \%$ & $34 \%$ & $(3: 2)$ \\
\hline & 6e: $\mathrm{R}=\mathrm{Et}, \mathrm{X}=\mathrm{NTs}$ & $60 \%$ & in mixtu & e $(3: 1)$ \\
\hline & 6f: $R={ }^{t} B u, X=N T s$ & 480 & in mixtu & e $(2: 1)$ \\
\hline
\end{tabular}

[a] Calculated in the NMR spectra of the crude

[b] Under conventional heating, $18 \mathrm{~h}$
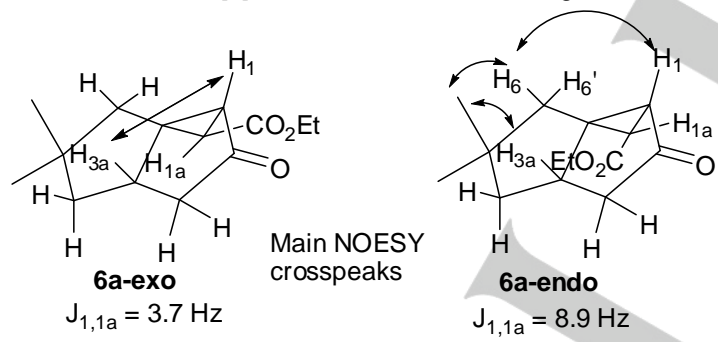

Scheme 3. Synthesis of products 6 .

\section{Conclusions}

We describe herein a microwave-mediated procedure for the cyclopropanation of $\alpha, \beta$-unsaturated ketones which reduces substantially the reaction time compared with previously reported results. This methodology is useful with different kinds of substrates. In particular we describe the synthesis of highly rigidified tricyclic compounds, including a precursor of pentalenene, an antibiotic produced by several species of Streptomyces, which has shown to be active against a large number of eukaryotic organisms. Using a microwave reactor provides greater control over reaction parameters, permitting a more efficient optimization of the reaction conditions.

\section{Experimental Section}

General: All reactions were carried out in a Biotage Initiator+ microwave reactor. Reaction progress was monitored using analytical thin-layer chromatography (TLC) on Merck silica gel 60 F-254 plate. Visualization was achieved by UV light (254 nm). NMR spectra were recorded on a Bruker spectrometer $\left(400 \mathrm{MHz}\right.$ for ${ }^{1} \mathrm{H}$, and $101 \mathrm{MHz}$ for ${ }^{13} \mathrm{C}$ ). Chemical shifts are reported in $\delta$ ppm referenced to chloroform- $d\left(\delta 7.26\right.$ for ${ }^{1} \mathrm{H}$ NMR and $\delta 77.00$ for ${ }^{13} \mathrm{C} N M R$ ). All the residues were purified by flash chromatography on silica gel. Bidimensional spectra (HMQC, HMBC, COSY, NOESY) were recorded for all compounds in order to carry out the assignation. Sulfonium salts were prepared following the procedures described in the literature. ${ }^{[14]}$

General procedure for the MW mediated cyclopropanation reaction: To a stirred solution of the corresponding salt (1.1 eq.) in $\mathrm{CHCl}_{3}(1 \mathrm{M})$ was added DBU (1.1 equiv). After 30 minutes, the corresponding substrate was added (1 equiv) and the reaction mixture was heated up to $70^{\circ} \mathrm{C}$ through microwave irradiation during $10 \mathrm{~min}$. The reaction mixture was then washed with $\mathrm{HCl} 1 \mathrm{M}(0.8 \mathrm{ml} /$ equiv), water $(2 \times 0.8 \mathrm{ml} /$ equiv) and brine $\left(2 \times 0.8 \mathrm{ml} /\right.$ equiv), dried over $\mathrm{MgSO}_{4}$, filtered and concentrated under vacuum to give the desired compounds.

Ethyl 2-oxobicyclo[3.1.0]hexane-6-carboxylate, 2a: From $150 \mathrm{mg}$ of cyclopent-2-enone $(1.82 \mathrm{mmol})$ and $458.3 \mathrm{mg}$ of salt $1 \mathrm{a}(2 \mathrm{mmol})$, a crude mixture of isomeric cyclopropanes (exo:endo) was obtained (12:1). After chromatography (Hexane/AcOEt, 9:1), $228 \mathrm{mg}$ (74\% yield) of pure

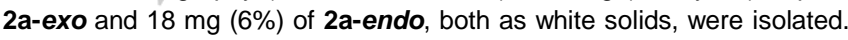
Using $510 \mathrm{mg}$ of salt $\mathbf{1 b}(2 \mathrm{mmol})$ gave a mixture of both isomers (exo:endo 10:1). After chromatography (Hexane/AcOEt, 9:1) $195 \mathrm{mg}$ (63\% yield) of pure $\mathbf{2 a - e x o}$ and $17 \mathrm{mg}(6 \%)$ of $\mathbf{2 a - e n d o , ~ b o t h ~ a s ~ w h i t e ~}$ solids, were isolated. Both isomers gave the same spectroscopical data as described previously. ${ }^{[5]}$

tert-Butyl 2-oxobicyclo[3.1.0]hexane-6-carboxylate, 2b: From $150 \mathrm{mg}$ of cyclopent-2-enone $(1.82 \mathrm{mmol})$ and $566.5 \mathrm{mg}$ of salt $1 \mathrm{c}(2 \mathrm{mmol})$, a crude mixture of isomeric (exo:endo) cyclopropanes was obtained (9:1). After chromatography (Hexane/AcOEt, 9:1), $271 \mathrm{mg}(76 \%$ yield) of pure $\mathbf{2 b}$-exo and $\mathbf{3 0} \mathrm{mg}(\mathbf{9} \%)$ of $\mathbf{2} \mathbf{b}$-endo, both as white solids, were isolated. Both isomers gave the same spectroscopical data as described previously. ${ }^{[5]}$

tert-Butyl 1-methyl-2-oxobicyclo[3.1.0]hexane-6-carboxylate, 2c From $150 \mathrm{mg}$ of 2-methylcyclopentenone $(1.56 \mathrm{mmol})$ and $488.24 \mathrm{mg}$ of salt $1 \mathrm{c}(1.71 \mathrm{mmol})$, a crude mixture of isomeric cyclopropanes was obtained (7:1). After chromatography (Hexane/AcOEt, 9:1), $190 \mathrm{mg}(58 \%$ yield) of pure 2c-exo (Rf: 0.31, Hexane/AcOEt, 9:1) and $52 \mathrm{mg}(16 \%)$ of a 1:1 mixture of both isomers, both as colorless oils, were isolated. Major isomer showed a $J_{\text {trans }}=3.9 \mathrm{~Hz}$ between $\mathrm{H} 6$ and $\mathrm{H} 5$ so it was assigned

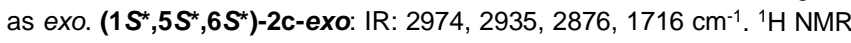
$\left(400 \mathrm{MHz}, \mathrm{CDCl}_{3}\right) \delta 2.42-2.40\left(\mathrm{~m}, 1 \mathrm{H}, \mathrm{CH}_{2} \mathrm{CH}\right), 2.15-2.00(\mathrm{~m}, 4 \mathrm{H}$, $\left.\mathrm{CH}_{2} \mathrm{CH}_{2}\right), 1.88\left(\mathrm{~d}, \mathrm{~J}=3.9 \mathrm{~Hz}, 1 \mathrm{H}, \mathrm{CHCO}_{2}\right), 1.46\left(\mathrm{~s}, 9 \mathrm{H}, \mathrm{C}\left(\mathrm{CH}_{3}\right)_{3}\right), 1.31$ (s, $\left.3 \mathrm{H}, \mathrm{CCH}_{3}\right) .{ }^{13} \mathrm{C}$ NMR $\left(101 \mathrm{MHz}, \mathrm{CDCl}_{3}\right) \delta 214.2\left(\mathrm{CH}_{2} \mathrm{C}=\mathrm{O}\right), 168.4\left(\mathrm{CO}_{2}\right.$

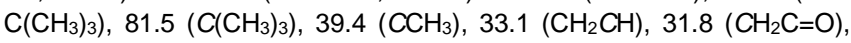

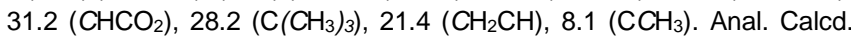
for $\mathrm{C}_{12} \mathrm{H}_{18} \mathrm{O}_{3}(210.27 \mathrm{~g} / \mathrm{mol}): \mathrm{C}, 68.5 ; \mathrm{H}, 8.6 \%$. Found: $\mathrm{C}, 68.7 ; \mathrm{H}, 8.4 \%$. $\left(\mathbf{1 S}^{*}, \mathbf{5} \mathrm{S}^{*}, \mathbf{6} \mathrm{R}^{*}\right)$-2c-endo (data taken from a diastereomeric enriched mixture): IR: 2974, 2935, 2876, $1716 \mathrm{~cm}^{-1} .{ }^{1} \mathrm{H}$ NMR $\left(400 \mathrm{MHz}, \mathrm{CDCl}_{3}\right) \delta$ $2.38\left(\mathrm{~m}, 1 \mathrm{H}, \mathrm{CH}_{2} \mathrm{CH}\right), 2.34-2.19\left(\mathrm{~m}, 4 \mathrm{H}, \mathrm{CH}_{2} \mathrm{C}=\mathrm{O}+\mathrm{CH}_{2} \mathrm{CHH}\right), 2.04(\mathrm{dd}$, 
$\left.\mathrm{J}=9.3,7.8 \mathrm{~Hz}, 1 \mathrm{H}, \mathrm{CH}_{2} \mathrm{CHH}\right), 1.44\left(\mathrm{~s}, 9 \mathrm{H}, \mathrm{C}\left(\mathrm{CH}_{3}\right)_{3}\right), 1.29\left(\mathrm{~s}, 3 \mathrm{H}, \mathrm{CCH}_{3}\right)$. ${ }^{13} \mathrm{C}$ NMR $\left(101 \mathrm{MHz}, \mathrm{CDCl}_{3}\right) \delta 214.2\left(\mathrm{CH}_{2} \mathrm{C}=\mathrm{O}\right), 168.9\left(\mathrm{CO}_{2} \mathrm{C}\left(\mathrm{CH}_{3}\right)_{3}\right)$, $81.5\left(\mathrm{C}_{\left.\left(\mathrm{CH}_{3}\right)_{3}\right),} 39.2\left(\mathrm{CCH}_{3}\right), 37.6\left(\mathrm{CH}_{2} \mathrm{C}=\mathrm{O}\right), 36.3\left(\mathrm{CH}_{2} \mathrm{CH}\right), 31.2\right.$ $\left(\mathrm{CHCO}_{2}\right), 28.1\left(\mathrm{C}_{\left.\left(\mathrm{CH}_{3}\right)_{3}\right),} 19.00\left(\mathrm{CH}_{2} \mathrm{CH}\right), 15.9\left(\mathrm{CCH}_{3}\right)\right.$.

\begin{abstract}
$\left(1 R^{*}, 5 R^{*}, 6 S^{*}\right)$-tert-Butyl 1-methyl-4-oxobicyclo[3.1.0]hexane-6carboxylate, 2d-endo: From $150 \mathrm{mg}$ of 3-methylcyclopentenone (1.56 $\mathrm{mmol}$ ) and $488.24 \mathrm{mg}$ of salt $1 \mathrm{c}(1.71 \mathrm{mmol})$, after chromatography (Hexane/AcOEt, 9:1), $69 \mathrm{mg}$ (21\% yield) of pure product (Rf: 0.10 , Hexane/AcOEt, 9:1). The product showed a $J_{\text {cis }}=9.4 \mathrm{~Hz}$ between $\mathrm{H} 6$ and $\mathrm{H} 5$ so it was assigned as endo $\left(1 S^{\star}, 5 S^{\star}, 6 S^{\star}\right)$. IR: $2978,2923,1728$ $\mathrm{cm}^{-1} .{ }^{1} \mathrm{H}$ NMR $\left(400 \mathrm{MHz}, \mathrm{CDCl}_{3}\right) \delta 2.44-2.25\left(\mathrm{~m}, 2 \mathrm{H}, \mathrm{CH}_{2} \mathrm{C}=\mathrm{O}\right), 2.25-$ $2.16\left(\mathrm{~m}, 1 \mathrm{H}, \mathrm{CHHCCH}_{3}\right), 2.10\left(\mathrm{~d}, \mathrm{~J}=9.4 \mathrm{~Hz}, 1 \mathrm{H}, \mathrm{CHCO}_{2}\right), 2.07-1.99$ $\left(\mathrm{m}, 1 \mathrm{H}, \mathrm{CHHCCH}_{3}\right), 1.97(\mathrm{~d}, \mathrm{~J}=9.4 \mathrm{~Hz}, 1 \mathrm{H}, \mathrm{CHC}=\mathrm{O}), 1.44(\mathrm{~s}, 9 \mathrm{H}$, $\left.\mathrm{C}\left(\mathrm{CH}_{3}\right)_{3}\right), 1.41\left(\mathrm{~s}, 3 \mathrm{H}, \mathrm{CCH}_{3}\right) .{ }^{13} \mathrm{C} \mathrm{NMR}\left(101 \mathrm{MHz}, \mathrm{CDCl}_{3}\right) \delta 213.4$ $\left(\mathrm{CH}_{2} \mathrm{C}=\mathrm{O}\right), \quad 168.7 \quad\left(\mathrm{CHCO}_{2}\right), \quad 81.7 \quad\left(\mathrm{C}_{\left.\left(\mathrm{CH}_{3}\right)_{3}\right),} 40.8(\mathrm{CHC}=\mathrm{O}), 38.4\right.$

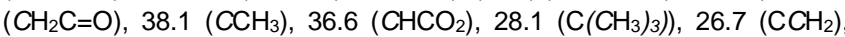
$23.0\left(\mathrm{CCH}_{3}\right)$. Anal. Calcd. for $\mathrm{C}_{12} \mathrm{H}_{18} \mathrm{O}_{3}(210.27 \mathrm{~g} / \mathrm{mol}): \mathrm{C}, 68.5 ; \mathrm{H}, 8.6 \%$. Found: C, 68.7; $\mathrm{H}, 8.8 \%$.
\end{abstract}

\section{$\left(1 R^{*}, 5 R^{*}, 6 S^{*}\right)$-Ethyl}

1-methyl-4-oxobicyclo[3.1.0]hexane-6carboxylate, 2e-endo: From $150 \mathrm{mg}$ of 3-methylcyclopentenone (1.56 $\mathrm{mmol})$ and $395 \mathrm{mg}$ of salt $1 \mathbf{a}(1.71 \mathrm{mmol})$, after chromatography (Hexane/AcOEt, 6:1), $59 \mathrm{mg}$ (22\% yield) of pure product (Rf: 0.10 , Hexane/AcOEt, 6:1). The product showed a $J_{\text {cis }}=9.5 \mathrm{~Hz}$ between $\mathrm{H} 6$ and $\mathrm{H} 5$ so it was assigned as $\left(1 \mathrm{~S}^{*}, 5 \mathrm{~S}^{\star}, 6 \mathrm{~S}^{\star}\right) .{ }^{1} \mathrm{H}$ NMR $\left(400 \mathrm{MHz}, \mathrm{CDCl}_{3}\right)$ $\delta 4.18-4.11\left(\mathrm{~m}, 2 \mathrm{H}, \mathrm{CH}_{2} \mathrm{CH}_{3}\right), 2.49-2.38(\mathrm{~m}, 1 \mathrm{H}, \mathrm{CH} H \mathrm{C}=\mathrm{O}), 2.38-$ $2.28(\mathrm{~m}, 1 \mathrm{H}, \mathrm{CHHC}=\mathrm{O}), 2.26-2.18(\mathrm{~m}, 1 \mathrm{H}, \mathrm{CH} H \mathrm{C}), 2.16(\mathrm{~d}, \mathrm{~J}=9.5 \mathrm{~Hz}$, $\left.1 \mathrm{H}, \mathrm{CHCO}_{2}\right), 2.10-2.01(\mathrm{~m}, 1 \mathrm{H}, \mathrm{CHHC}), 2.05(\mathrm{~d}, \mathrm{~J}=9.5 \mathrm{~Hz}, 1 \mathrm{H}$, $\mathrm{CHC}=\mathrm{O}), 1.44\left(\mathrm{~s}, 3 \mathrm{H}, \mathrm{CCH}_{3}\right), 1.29-1.24\left(\mathrm{t}, \mathrm{J}=7.1 \mathrm{~Hz}, 3 \mathrm{H}, \mathrm{CH}_{2} \mathrm{CH}_{3}\right)$. ${ }^{13} \mathrm{C}$ NMR $\left(101 \mathrm{MHz}, \mathrm{CDCl}_{3}\right) \delta 213.3\left(\mathrm{CH}_{2} \mathrm{C}=\mathrm{O}\right), 169.6\left(\mathrm{CO}_{2} \mathrm{CH}_{2}\right), 61.2$ $\left(\mathrm{CO}_{2} \mathrm{CH}_{2}\right), 41.1(\mathrm{CHC}=\mathrm{O}), 38.6\left(\mathrm{CH}_{2} \mathrm{C}=\mathrm{O}\right), 38.5\left(\mathrm{CCH}_{3}\right), 35.3\left(\mathrm{CHCO}_{2}\right)$, $26.8\left(\mathrm{CH}_{2} \mathrm{C}\right), 23.0\left(\mathrm{CCH}_{3}\right), 14.2\left(\mathrm{CH}_{2} \mathrm{CH}_{3}\right)$. Anal. Calcd. for $\mathrm{C}_{10} \mathrm{H}_{14} \mathrm{O}_{3}$ (182.22 g/mol): C, 65.9; H, 7.7 \%. Found: C, 65.6; H, $7.5 \%$.

(15*,5R*,6S*)-6-(4-methoxybenzoyl)bicyclo[3.1.0]hexan-2-one, 2fexo: From $150 \mathrm{mg}$ of cyclopentenone $(1.83 \mathrm{mmol})$ and $586.2 \mathrm{mg}$ of salt 1d $(2.03 \mathrm{mmol}$ ), after chromatography (Hexane/AcOEt, 9:1), $355 \mathrm{mg}$ (91\% yield) of pure product were obtained (Rf: 0.05, Hexane/AcOEt, 9:1). The product showed a $J_{\text {trans }}=2.5 \mathrm{~Hz}$ between $\mathrm{H} 6$ and $\mathrm{H} 1$ and a $J_{\text {trans }}=$ $3.4 \mathrm{~Hz}$ between $\mathrm{H} 6$ and $\mathrm{H} 5$ so it was assigned as $\left(1 S^{*}, 5 S^{\star}, 6 S^{\star}\right)$. IR: 2949, 2911, 2840, 1727, 1660, $1590 \mathrm{~cm}^{-1} .{ }^{1} \mathrm{H} \mathrm{NMR}\left(400 \mathrm{MHz}, \mathrm{CDCl}_{3}\right) \delta$ $7.99-7.94(\mathrm{~m}, 2 \mathrm{H}, \mathrm{CHCC}=\mathrm{O}), 6.99-6.94\left(\mathrm{~m}, 2 \mathrm{H}, \mathrm{CHCOCH}_{3}\right), 3.89(\mathrm{~s}$, $\left.3 \mathrm{H}, \mathrm{OCH}_{3}\right), 2.95$ (dd, $\mathrm{J}=3.4,2.5 \mathrm{~Hz}, 1 \mathrm{H}, \mathrm{CHC}=\mathrm{OAr}$ ), 2.66 (td, $\mathrm{J}=5.2$, $\left.3.4 \mathrm{~Hz}, 1 \mathrm{H}, \mathrm{CH}_{2} \mathrm{CH}\right), 2.48\left(\mathrm{dd}, \mathrm{J}=5.5,2.5 \mathrm{~Hz}, 1 \mathrm{H}, \mathrm{CH}_{2} \mathrm{C}=\mathrm{OCH}\right), 2.39-$ $2.25(\mathrm{~m}, 1 \mathrm{H}, \mathrm{CHHCH}), 2.24-2.18\left(\mathrm{~m}, 3 \mathrm{H}, \mathrm{CHHCH}+\mathrm{CH}_{2} \mathrm{C}=\mathrm{O}\right) .{ }^{13} \mathrm{C}$ NMR $\left(101 \mathrm{MHz}, \mathrm{CDCl}_{3}\right) \delta 212.6\left(\mathrm{CH}_{2} \mathrm{C}=\mathrm{O}\right), 193.6(\mathrm{CHC}=\mathrm{OC}), 163.8(\mathrm{Ar})$ 130.5 ( $\mathrm{Ar}), 129.9(\mathrm{Ar}), 113.9(\mathrm{Ar}), 55.5\left(\mathrm{OCH}_{3}\right), 38.2\left(\mathrm{CH}_{2} \mathrm{C}=\mathrm{OCH}\right), 32.5$ $\left(\mathrm{CH}_{2} \mathrm{C}=\mathrm{O}\right), 31.7\left(\mathrm{CH}_{2} \mathrm{CH}\right), 29.8(\mathrm{CHC}=\mathrm{OAr}), 22.8\left(\mathrm{CH}_{2} \mathrm{CH}\right)$. Anal. Calcd. for $\mathrm{C}_{14} \mathrm{H}_{14} \mathrm{O}_{3}(230.26 \mathrm{~g} / \mathrm{mol}): \mathrm{C}, 73.0 ; \mathrm{H}, 6.1 \%$. Found: $\mathrm{C}, 72.8 ; \mathrm{H}, 6.4 \%$.

Diethyl 1-(2,2-bis(ethoxycarbonyl)pent-4-en-1-yl)cyclopropane-1,2dicarboxylate, 4: From $300 \mathrm{mg}$ of triethyl hepta-1,6-diene-2,4,4tricarboxylate $^{[15]}(0.96 \mathrm{mmol})$ and $260 \mathrm{mg}$ of salt $1 \mathrm{a}(1.01 \mathrm{mmol})$, a crude mixture of isomeric cyclopropanes was obtained (6:1). After chromatography (Hexane/AcOEt, 19:1), $224 \mathrm{mg}$ (59\% yield) of pure major isomer (Rf: 0.11, Hexane/AcOEt, 19:1) and $42 \mathrm{mg}$ (11\%) of minor isomer (Rf: 0.03, Hexane/AcOEt, 19:1), both as colorless oils, were isolated. Major isomer showed a ${ }^{4} \mathrm{~J}=1.2 \mathrm{~Hz}$ between $\mathrm{H}^{\prime}$ and $\mathrm{CH}_{2}$ and H3' showed a $J_{\text {cis }}=8.7 \mathrm{~Hz}$ with H2. NOESY spectrum showed cross peaks of $\mathrm{H} 3$ with $\mathrm{H} 2$ (strong) and with both $\mathrm{CH}_{2}$ protons (weak). Thus, this isomer was assigned as $\left(1 R^{\star}, 2 S^{\star}\right)$. IR: $2984,2915,1730,1640 \mathrm{~cm}^{-1}$. ${ }^{1} \mathrm{H}$ NMR $\left(400 \mathrm{MHz}, \mathrm{CDCl}_{3}\right) \delta 5.79$ (ddt, $\mathrm{J}=17.3,10.2,7.2 \mathrm{~Hz}, 1 \mathrm{H}$, $\left.\mathrm{CH}_{2}=\mathrm{CH}\right), 5.11-5.03\left(\mathrm{~m}, 2 \mathrm{H}, \mathrm{CH}_{2}=\mathrm{CH}\right), 4.26-4.00\left(\mathrm{~m}, 8 \mathrm{H}, \mathrm{CO}_{2} \mathrm{CH}_{2}\right)$, $2.73\left(\mathrm{dt}, \mathrm{J}=14.2,1.3 \mathrm{~Hz}, 1 \mathrm{H}, \mathrm{CH}_{2} \mathrm{CH}=\mathrm{CH}_{2}\right), 2.71(\mathrm{dd}, \mathrm{J}=15.2,1.2 \mathrm{~Hz}$, $1 \mathrm{H}, \mathrm{CCHHC}$ ), 2.58 (ddt, $\mathrm{J}=14.2,7.2,1.3 \mathrm{~Hz}, 1 \mathrm{H}, \mathrm{CH}_{2} \mathrm{CH}=\mathrm{CH}_{2}$ ), 2.42 (d, $\mathrm{J}=15.2 \mathrm{~Hz}, 1 \mathrm{H}, \mathrm{CCHHC}), 2.06\left(\mathrm{dd}, \mathrm{J}=8.7,6.7 \mathrm{~Hz}, 1 \mathrm{H}, \mathrm{CHCO}_{2}\right), 1.76$ (ddd, J = 8.7, 4.8, $1.2 \mathrm{~Hz}, 1 \mathrm{H}, \mathrm{CHHCHCO}_{2}$ ), 1.53 (dd, J = 6.7, $4.8 \mathrm{~Hz}, 1 \mathrm{H}$, $\mathrm{CH}_{\mathrm{CHCO}}$ ), $1.31-1.21\left(\mathrm{~m}, 12 \mathrm{H}, \mathrm{CH}_{2} \mathrm{CH}_{3}\right) \cdot{ }^{13} \mathrm{C} \mathrm{NMR}(101 \mathrm{MHz}$, $\left.\mathrm{CDCl}_{3}\right) \delta 172.2\left(\mathrm{CO}_{2} \mathrm{CH}_{2} \mathrm{CH}_{3}\right), 171.0\left(\mathrm{CO}_{2} \mathrm{CH}_{2} \mathrm{CH}_{3}\right), 170.8\left(\mathrm{CO}_{2} \mathrm{CH}_{2} \mathrm{CH}_{3}\right)$, $169.9\left(\mathrm{CO}_{2} \mathrm{CH}_{2} \mathrm{CH}_{3}\right), \quad 132.9 \quad\left(\mathrm{CH}_{2}=\mathrm{CH}\right), \quad 118.7 \quad\left(\mathrm{CH}_{2}=\mathrm{CH}\right), \quad 61.4$ $\left(\mathrm{CO}_{2} \mathrm{CH}_{2} \mathrm{CH}_{3}\right), \quad 61.2 \quad\left(\mathrm{CO}_{2} \mathrm{CH}_{2} \mathrm{CH}_{3}\right), \quad 61.2 \quad\left(\mathrm{CO}_{2} \mathrm{CH}_{2} \mathrm{CH}_{3}\right), \quad 61.1$ $\left(\mathrm{CO}_{2} \mathrm{CH}_{2} \mathrm{CH}_{3}\right), 57.2(\mathrm{C}=\mathrm{OCC}=\mathrm{O}), 39.5\left(\mathrm{CH}_{2} \mathrm{C}=\mathrm{CH}_{2}\right), 29.7\left(\mathrm{CH}_{2} \mathrm{CCH}_{2}\right)$, $29.3\left(\mathrm{CCH}_{2} \mathrm{C}\right), 28.3(\mathrm{CCH}), 18.0\left(\mathrm{CH}_{2} \mathrm{CHCO}_{2}\right), 14.2\left(\mathrm{CO}_{2} \mathrm{CH}_{2} \mathrm{CH}_{3}\right), 14.0$ $\left(\mathrm{CO}_{2} \mathrm{CH}_{2} \mathrm{CH}_{3}\right), 13.9\left(\mathrm{CO}_{2} \mathrm{CH}_{2} \mathrm{CH}_{3}\right), 13.8\left(\mathrm{CO}_{2} \mathrm{CH}_{2} \mathrm{CH}_{3}\right)$. Anal. Calcd. for $\mathrm{C}_{20} \mathrm{H}_{30} \mathrm{O}_{8}(398.45 \mathrm{~g} / \mathrm{mol}): \mathrm{C}, 60.3 ; \mathrm{H}, 7.6 \%$. Found: C, 60.1; H, $7.3 \%$. Minor isomer was assigned as $\left(1 S^{\star}, 2 S^{\star}\right)$ : IR: 2984, 2940, 2911, 1730, $1635 \mathrm{~cm}^{-1} .{ }^{1} \mathrm{H}$ NMR $\left(400 \mathrm{MHz}, \mathrm{CDCl}_{3}\right) \delta 5.66$ (ddt, $J=17.3,10.2,7.3 \mathrm{~Hz}$, $\left.1 \mathrm{H}, \mathrm{CH}_{2}=\mathrm{CH}\right), 5.14-5.02\left(\mathrm{~m}, 2 \mathrm{H}, \mathrm{CH}_{2}=\mathrm{CH}\right), 4.26-3.96(\mathrm{~m}, 8 \mathrm{H}$, $\mathrm{CO}_{2} \mathrm{CH}_{2} \mathrm{CH}_{3}$ ), 2.89 (d, J = $15.1 \mathrm{~Hz}, 1 \mathrm{H}, \mathrm{CHHC}$ ), 2.73 (qd, $\mathrm{J}=14.6,7.3$ $\left.\mathrm{Hz}, 2 \mathrm{H}, \mathrm{CCH}_{2} \mathrm{CH}=\mathrm{CH}_{2}\right), 1.86$ (d, J $\left.=15.0 \mathrm{~Hz}, 1 \mathrm{H}, \mathrm{CCHHC}\right), 1.84-1.79$ $\left(\mathrm{m}, 2 \mathrm{H}, \mathrm{CHCO}_{2}+\mathrm{CHHCHCO}_{2}\right), 1.30-1.17\left(\mathrm{~m}, 13 \mathrm{H}, \mathrm{CHHCHCO}_{2}+\right.$ $\left.\mathrm{CO}_{2} \mathrm{CH}_{2} \mathrm{CH}_{3}\right) .{ }^{13} \mathrm{C}$ NMR $\left(101 \mathrm{MHz}, \mathrm{CDCl}_{3}\right) \delta 170.6\left(\mathrm{CO}_{2} \mathrm{CH}_{2} \mathrm{CH}_{3}\right), 170.5$ $\left(\mathrm{CO}_{2} \mathrm{CH}_{2} \mathrm{CH}_{3}\right), \quad 170.2\left(\mathrm{CO}_{2} \mathrm{CH}_{2} \mathrm{CH}_{3}\right), \quad 170.0 \quad\left(\mathrm{CO}_{2} \mathrm{CH}_{2} \mathrm{CH}_{3}\right), \quad 132.5$ $\left(\mathrm{CH}_{2}=\mathrm{CH}\right), 119.1\left(\mathrm{CH}_{2}=\mathrm{CH}\right), 61.4\left(\mathrm{CO}_{2} \mathrm{CH}_{2} \mathrm{CH}_{3}\right), 61.4\left(\mathrm{CO}_{2} \mathrm{CH}_{2} \mathrm{CH}_{3}\right)$, $61.1\left(\mathrm{CO}_{2} \mathrm{CH}_{2} \mathrm{CH}_{3}\right), 60.9\left(\mathrm{CO}_{2} \mathrm{CH}_{2} \mathrm{CH}_{3}\right), 57.4\left(\mathrm{CO}_{2} \mathrm{CCO}_{2}\right), 37.9\left(\mathrm{CCH}_{2} \mathrm{C}\right)$, $37.3\left(\mathrm{CCH}_{2} \mathrm{CH}=\mathrm{CH}_{2}\right), 31.0\left(\mathrm{CH}_{2} \mathrm{CCH}\right), 28.1(\mathrm{CCH}), 18.1\left(\mathrm{CH}_{2} \mathrm{CHCO}_{2}\right)$, $14.2\left(\mathrm{CO}_{2} \mathrm{CH}_{2} \mathrm{CH}_{3}\right), \quad 13.9\left(\mathrm{CO}_{2} \mathrm{CH}_{2} \mathrm{CH}_{3}\right), \quad 13.9\left(\mathrm{CO}_{2} \mathrm{CH}_{2} \mathrm{CH}_{3}\right), \quad 13.9$ $\left(\mathrm{CO}_{2} \mathrm{CH}_{2} \mathrm{CH}_{3}\right)$. Anal. Calcd. for $\mathrm{C}_{20} \mathrm{H}_{30} \mathrm{O}_{8}(398.45 \mathrm{~g} / \mathrm{mol}): \mathrm{C}, 60.3 ; \mathrm{H}$, $7.6 \%$. Found: C, $60.4 ; \mathrm{H}, 7.4 \%$.

Ethyl 5,5-dimethyl-2-oxooctahydrocyclopropa[c]pentalene-1carboxylate, $\mathbf{6 a}$ : From $150 \mathrm{mg}$ of $\mathbf{5 a}^{[12]}(1.00 \mathrm{mmol})$ and $252.05 \mathrm{mg}$ of salt $1 \mathrm{a}(1.10 \mathrm{mmol})$, a crude mixture of isomeric cyclopropanes was obtained (1:1). After chromatography (Hexane/AcOEt, 14:1), $73 \mathrm{mg}(31 \%$ yield) of a colorless oil (Rf: 0.22; Hexane/AcOEt, 14:1) was obtained. This isomer was assigned as $6 \mathbf{a}-\mathbf{e x o}\left(1 \mathrm{~S}^{*}, 1 \mathrm{aS}^{*}, 3 \mathrm{aS}^{*}, 6 \mathrm{aS}^{\star}\right)$ based on: Coupling constant of $J_{\text {trans }}=3.7 \mathrm{~Hz}$ between $\mathrm{H} 1 \mathrm{a}$ and $\mathrm{H} 1$. NOESY spectrum showed cross peaks of $\mathrm{H} 1 \mathrm{a}$ with $\mathrm{H} 3 \mathrm{a} .68 \mathrm{mg}$, (29\%) of another fraction containing a (13:1) mixture of the second isomer and the starting material was isolated Rf: 0.18 (Hexane/AcOEt, 14:1). Data for second isomer are given from this mixture. Configuration was assigned as $6 \mathbf{a}-$ endo $\left(1 \mathrm{R}^{*}, 1 \mathrm{aS}^{*}, 3 \mathrm{aS}^{*}, 6 \mathrm{aS}^{*}\right)$ based on: Coupling constant of $J_{\text {cis }}=8.9$ $\mathrm{Hz}$ between $\mathrm{H} 1 \mathrm{a}$ and $\mathrm{H} 1$. NOESY spectrum showed cross peaks of $\mathrm{H} 1 \mathrm{a}$ with one $\mathrm{H} 6$ which showed cross peaks with 1 methyl group. This very same methyl group showed cross peaks with $\mathrm{H} 3 \mathrm{a}$. In addition, H1 showed cross peaks with the other $\mathrm{H} 6$ proton. This agrees with the configuration presented according to 3D models. 6a-exo. IR: 2956, 2936, 2867, $1726 \mathrm{~cm}^{-1}$. ${ }^{1} \mathrm{H}$ NMR $\left(400 \mathrm{MHz}, \mathrm{CDCl}_{3}\right) \delta 4.24-4.07(\mathrm{~m}, 2 \mathrm{H}$, $\left.\mathrm{CH}_{2} \mathrm{CH}_{3}\right), 2.73-2.64\left(\mathrm{~m}, 1 \mathrm{H}, \mathrm{CH}_{2} \mathrm{CHCH}_{2}\right), 2.23(\mathrm{dd}, \mathrm{J}=18.7,7.8 \mathrm{~Hz}, 1 \mathrm{H}$, $\mathrm{CH} H \mathrm{C}=\mathrm{O}), 2.21\left(\mathrm{~d}, \mathrm{~J}=3.1 \mathrm{~Hz}, 1 \mathrm{H}, \mathrm{CHCO}_{2}\right), 2.17(\mathrm{~d}, \mathrm{~J}=3.1 \mathrm{~Hz}, 1 \mathrm{H}$, $\mathrm{CHCHC}=\mathrm{O}), 2.02(\mathrm{dt}, \mathrm{J}=19.1,1.5 \mathrm{~Hz}, 1 \mathrm{H}, \mathrm{CHHC}=\mathrm{O}), 1.91-1.79(\mathrm{~m}$, $\left.3 \mathrm{H}, \mathrm{CCH}_{2} \mathrm{C}+\mathrm{CCHHCH}\right), 1.32-1.24(\mathrm{~m}, 1 \mathrm{H}, \mathrm{CCHHCH}), 1.27(\mathrm{t}, \mathrm{J}=7.1$ $\left.\mathrm{Hz}, 3 \mathrm{H}, \mathrm{CH}_{2} \mathrm{CH}_{3}\right), 1.12\left(\mathrm{~s}, 3 \mathrm{H}, \mathrm{CCH}_{3}\right), 1.09\left(\mathrm{~s}, 3 \mathrm{H}, \mathrm{CCH}_{3}\right) .{ }^{13} \mathrm{C}$ NMR $(101$ $\left.\mathrm{MHz}, \mathrm{CDCl}_{3}\right) \delta 212.1\left(\mathrm{CH}_{2} \mathrm{C}=\mathrm{O}\right), 169.5\left(\mathrm{CO}_{2} \mathrm{CH}_{2}\right), 61.1\left(\mathrm{CH}_{2} \mathrm{CH}_{3}\right), 48.6$ $\left(\mathrm{CCH}_{2} \mathrm{CH}\right), 47.7 \quad\left(\mathrm{CH}_{2} \mathrm{CCH}\right), 42.0 \quad\left(\mathrm{CHCO}_{2}\right), 41.7 \quad\left(\mathrm{CCH}_{2} \mathrm{C}\right), 40.7$ $\left(\mathrm{CH}_{2} \mathrm{CHCH}_{2}\right), 40.0\left(\mathrm{CH}_{2} \mathrm{C}=\mathrm{O}\right), 39.0\left(\mathrm{C}_{\left.\left(\mathrm{CH}_{3}\right)_{2}\right)}\right), 31.0(\mathrm{CHCHC}=\mathrm{O}), 30.2$

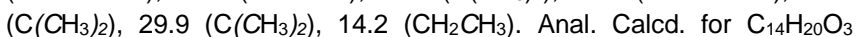
(236.31 g/mol): C, 71.2; H, $8.5 \%$. Found: C, 71.4; H, $8.8 \%$. 6a-endo: IR: 2956, 2936, 2867, $1726 \mathrm{~cm}^{-1} .{ }^{1} \mathrm{H}$ NMR $\left(400 \mathrm{MHz}, \mathrm{CDCl}_{3}\right) \delta 4.21-4.07$ (m, $\left.2 \mathrm{H}, \mathrm{CH}_{2} \mathrm{CH}_{3}\right), 2.79-2.67\left(\mathrm{~m}, 2 \mathrm{H}, \mathrm{CH} H \mathrm{C}=\mathrm{O}+\mathrm{CH}_{2} \mathrm{CHCH}_{2}\right), 2.38(\mathrm{~d}, \mathrm{~J}$ $=9.0 \mathrm{~Hz}, 1 \mathrm{H}, \mathrm{CHCHC}=\mathrm{O}), 2.15-2.06(\mathrm{~m}, 1 \mathrm{H}, \mathrm{CHHC}=\mathrm{O}), 2.12(\mathrm{~d}, \mathrm{~J}=$ $8.8 \mathrm{~Hz}, 1 \mathrm{H}, \mathrm{CHCO}_{2}$ ), 1.98 (ddd, $\mathrm{J}=12.5,8.0,1.3 \mathrm{~Hz}, 1 \mathrm{H}, \mathrm{CCHHCH}$ ), $1.86(\mathrm{~d}, \mathrm{~J}=13.3 \mathrm{~Hz}, 1 \mathrm{H}, \mathrm{CCHHC}), 1.64(\mathrm{~d}, \mathrm{~J}=13.3 \mathrm{~Hz}, 1 \mathrm{H}, \mathrm{CCHHC})$, 1.41 (dd, $J=12.7,8.4 \mathrm{~Hz}, 1 \mathrm{H}, \mathrm{CCHHCH}), 1.27(\mathrm{t}, J=7.1 \mathrm{~Hz}, 3 \mathrm{H}$, $\left.\mathrm{CH}_{2} \mathrm{CH}_{3}\right), 1.12\left(\mathrm{~s}, 3 \mathrm{H}, \mathrm{C}\left(\mathrm{CH}_{3}\right)_{2}\right), 1.09\left(\mathrm{~s}, 3 \mathrm{H}, \mathrm{C}\left(\mathrm{CH}_{3}\right)_{2}\right) .{ }^{13} \mathrm{C} \mathrm{NMR}(101$ $\left.\mathrm{MHz}, \mathrm{CDCl}_{3}\right) \delta 213.7\left(\mathrm{CH}_{2} \mathrm{C}=\mathrm{O}\right), 170.1\left(\mathrm{CO}_{2} \mathrm{CH}_{2}\right), 61.2\left(\mathrm{CH}_{2} \mathrm{CH}_{3}\right), 49.8$ $\left(\mathrm{CH}_{2} \mathrm{CCH}\right), 49.7\left(\mathrm{CCH}_{2} \mathrm{CH}\right), 47.7\left(\mathrm{CH}_{2} \mathrm{C}=\mathrm{O}\right), 46.2\left(\mathrm{CCH}_{2} \mathrm{C}\right), 41.9$ 
$\left(\mathrm{CHCO}_{2}\right), 40.6\left(\mathrm{C}\left(\mathrm{CH}_{3}\right)_{2}\right), 37.7\left(\mathrm{CH}_{2} \mathrm{CHCH}_{2}\right), 33.8(\mathrm{CHCHC}=\mathrm{O}), 29.0$ $\left(\mathrm{C}\left(\mathrm{CH}_{3}\right)_{2}\right), 28.6\left(\mathrm{C}\left(\mathrm{CH}_{3}\right)_{2}\right), 14.2\left(\mathrm{CH}_{2} \mathrm{CH}_{3}\right)$

tert-Butyl 5,5-dimethyl-2-oxooctahydrocyclopropa[c]pentalene-1carboxylate, $\mathbf{6 b}$ : From $150 \mathrm{mg}$ of $\mathbf{5 a}^{[12]}(1.00 \mathrm{mmol})$ and $311.54 \mathrm{mg}$ of salt 1c $(1.10 \mathrm{mmol})$, a crude mixture of isomeric cyclopropanes was obtained (3:2). After chromatography (Hexane/AcOEt, 14:1), $77 \mathrm{mg}$ (29\% yield) of isomer as a colorless oil (Rf: 0.32; Hexane/AcOEt, 14:1). This isomer was assigned as $6 \mathbf{b}$-exo $\left(1 \mathrm{~S}^{*}, 1 \mathrm{aS}^{*}, 3 \mathrm{aS} \mathrm{S}^{*}, 6 \mathrm{aS^{* }}\right)$ based on: Coupling constant of $J_{\text {trans }}=2.9 \mathrm{~Hz}$ between $\mathrm{H} 1 \mathrm{a}$ and $\mathrm{H} 1$. NOESY spectrum showed cross peaks of $\mathrm{H} 1 \mathrm{a}$ with $\mathrm{H} 3 \mathrm{a} .42 \mathrm{mg}$, (16\%) of another fraction containing a (1:13 exo:endo) mixture Rf: 0.28 (Hexane/AcOEt, 14:1). Data for second isomer are given from this mixture. Configuration was assigned as $6 \mathrm{~b}$-endo $\left(1 \mathrm{R}^{*}, 1 \mathrm{aS}^{\star}, 3 \mathrm{aS}^{*}, 6 \mathrm{aS} \mathrm{S}^{\star}\right)$ based on: Coupling constant of $J_{\text {cis }}=9.0 \mathrm{~Hz}$ between $\mathrm{H} 1 \mathrm{a}$ and $\mathrm{H} 1$. NOESY spectrum showed cross peaks of $\mathrm{H} 1 \mathrm{a}$ with one $\mathrm{H} 6$ which showed cross peaks with 1 methyl group. This same methyl group showed cross peaks with $\mathrm{H} 3 \mathrm{a}$. In addition $\mathrm{H} 1$ showed cross peaks with the other $\mathrm{H} 6$ proton. This agrees with the conformation presented according to 3D models. 6b-exo: IR: 2956, 2867 2258, $1722 \mathrm{~cm}^{-1} .{ }^{1} \mathrm{H}$ NMR $\left(400 \mathrm{MHz}, \mathrm{CDCl}_{3}\right) \delta 2.73-2.64(\mathrm{~m}, 1 \mathrm{H}$, $\mathrm{CH}_{2} \mathrm{CHCH}_{2}$ ), 2.21 (dd, $\mathrm{J}=19.3,7.8 \mathrm{~Hz}, 1 \mathrm{H}, \mathrm{CHHC}=0$ ), 2.16 (d, $\mathrm{J}=2.9$ $\left.\mathrm{Hz}, 1 \mathrm{H}, \mathrm{CHCO}_{2}\right), 2.09$ (d, $\left.\mathrm{J}=2.9 \mathrm{~Hz}, 1 \mathrm{H}, \mathrm{CHCHC}=0\right), 1.99(\mathrm{dt}, \mathrm{J}=19.2$, $1.4 \mathrm{~Hz}, 1 \mathrm{H}, \mathrm{CHHC}=\mathrm{O}), 1.89-1.78\left(\mathrm{~m}, 3 \mathrm{H}, \mathrm{CCH}_{2} \mathrm{C}+\mathrm{CCHHCH}\right), 1.45$ (s, $\left.9 \mathrm{H}, \mathrm{C}\left(\mathrm{CH}_{3}\right)_{3}\right), 1.27$ (dd, $\left.\mathrm{J}=12.6,11.2 \mathrm{~Hz}, 1 \mathrm{H}, \mathrm{CCHHCH}\right), 1.12(\mathrm{~s}, 3 \mathrm{H}$, $\left.\mathrm{C}\left(\mathrm{CH}_{3}\right)_{2}\right), 1.09\left(\mathrm{~s}, 3 \mathrm{H}, \mathrm{C}\left(\mathrm{CH}_{3}\right)_{2}\right) .{ }^{13} \mathrm{C} \mathrm{NMR}\left(101 \mathrm{MHz}, \mathrm{CDCl}_{3}\right) \delta 212.5$ $\left(\mathrm{CH}_{2} \mathrm{C}=\mathrm{O}\right), \quad 168.5\left(\mathrm{CO}_{2} \mathrm{CH}_{2}\right), 81.6\left(\mathrm{C}_{\left.\left(\mathrm{CH}_{3}\right)_{3}\right),} 48.6\left(\mathrm{CCH}_{2} \mathrm{CH}\right), 47.6\right.$ $\left(\mathrm{CH}_{2} \mathrm{C}(\mathrm{CH})_{3}\right), 41.9\left(\mathrm{CHCO}_{2}\right), 41.8\left(\mathrm{CCH}_{2} \mathrm{C}\right), 40.7\left(\mathrm{CH}_{2} \mathrm{CHCH}_{2}\right), 40.0$ $\left(\mathrm{CH}_{2} \mathrm{C}=\mathrm{O}\right), 38.9\left(\mathrm{C}\left(\mathrm{CH}_{3}\right)_{2}\right), 32.1(\mathrm{CHCHC}=\mathrm{O}), 30.3\left(\mathrm{C}\left(\mathrm{CH}_{3}\right)_{2}\right), 29.9$ $\left(\mathrm{C}\left(\mathrm{CH}_{3}\right)_{2}\right), 28.1\left(\mathrm{C}\left(\mathrm{CH}_{3}\right)_{3}\right)$. Anal. Calcd. for $\mathrm{C}_{14} \mathrm{H}_{24} \mathrm{O}_{3}(264.36 \mathrm{~g} / \mathrm{mol}): \mathrm{C}$, 72.7; H, 9.2 \%. Found: C, 73.0; H, 9.4 \%. 6b-endo: IR: 2956, 2867, 2258 $1722 \mathrm{~cm}^{-1}$. ${ }^{1} \mathrm{H}$ NMR (400 MHz, $\left.\mathrm{CDCl}_{3}\right) \delta 2.74-2.64\left(\mathrm{~m}, 2 \mathrm{H}, \mathrm{CH}_{2} \mathrm{CHCH}_{2}\right.$ $+\mathrm{CHHC}=\mathrm{O}), 2.33(\mathrm{~d}, \mathrm{~J}=9.0 \mathrm{~Hz}, 1 \mathrm{H}, \mathrm{CHCHC}=\mathrm{O}), 2.16-2.01(\mathrm{~m}, 1 \mathrm{H}$, $\mathrm{CH} H \mathrm{C}=\mathrm{O}), 2.05\left(\mathrm{~d}, \mathrm{~J}=9.1 \mathrm{~Hz}, 1 \mathrm{H}, \mathrm{CHCO}_{2}\right), 1.97$ (ddd, $\mathrm{J}=12.5,7.9,1.2$ $\mathrm{Hz}, 1 \mathrm{H}, \mathrm{CCHHCH}), 1.84(\mathrm{~d}, \mathrm{~J}=12.8 \mathrm{~Hz}, 1 \mathrm{H}, \mathrm{CCH} H \mathrm{C}), 1.62$ (d, J = 13.3 $\mathrm{Hz}, 1 \mathrm{H}, \mathrm{CHHC}$ ), $1.44\left(\mathrm{~s}, 9 \mathrm{H}, \mathrm{C}\left(\mathrm{CH}_{3}\right)_{3}\right), 1.40$ (dd, $\mathrm{J}=12.7,8.4 \mathrm{~Hz}, 1 \mathrm{H}$, $\mathrm{CCHHCH}), 1.11\left(\mathrm{~s}, 3 \mathrm{H}, \mathrm{C}\left(\mathrm{CH}_{3}\right)_{2}\right), 1.09$ (s, 3H, C(CH$\left.)_{2}\right) .{ }^{13} \mathrm{C} \mathrm{NMR}(101$ $\left.\mathrm{MHz}, \mathrm{CDCl}_{3}\right) \delta 213.8\left(\mathrm{CH}_{2} \mathrm{C}=\mathrm{O}\right), 169.2\left(\mathrm{CO}_{2} \mathrm{CH}_{2}\right), 81.7\left(\mathrm{C}_{\left.\left(\mathrm{CH}_{3}\right)_{3}\right), 49.7}\right.$ $\left(\mathrm{CCH}_{2} \mathrm{CH}\right), 49.5\left(\mathrm{CH}_{2} \mathrm{CCH}\right), 47.7\left(\mathrm{CH}_{2} \mathrm{C}=\mathrm{O}\right), 46.2\left(\mathrm{CCH}_{2} \mathrm{C}\right), 41.6$ $\left(\mathrm{CHCO}_{2}\right), 40.6\left(\mathrm{C}\left(\mathrm{CH}_{3}\right)_{2}\right), 37.6\left(\mathrm{CH}_{2} \mathrm{CHCH}_{2}\right), 35.3(\mathrm{CHCHC}=\mathrm{O}), 29.0$ $\left(\mathrm{C}\left(\mathrm{CH}_{3}\right)_{2}\right), 28.5\left(\mathrm{C}\left(\mathrm{CH}_{3}\right)_{2}\right), 28.1\left(\mathrm{C}\left(\mathrm{CH}_{3}\right)_{3}\right)$

\section{Triethyl 2-oxohexahydrocyclopropa[c]pentalene-1,5,5(6H)-} tricarboxylate, $6 c$ : From $350 \mathrm{mg}$ of diethyl $\mathbf{5 b}^{[12]}(1.31 \mathrm{mmol})$ and 330 mg of salt 1a (1.44 mmol), a crude mixture of isomeric cyclopropanes was obtained (5:2). After chromatography (Hexane/AcOEt, 6:1), $195 \mathrm{mg}$ (42\% yield) of a colorless oil (Rf: 0.20; Hexane/AcOEt, 6:1) were obtained. This isomer was assigned as 6c-exo $\left(1 \mathrm{~S}^{*}, 1 \mathrm{aS}^{*}, 3 \mathrm{aS}^{*}, 6 \mathrm{aS}^{*}\right)$ based on: Coupling constant of $J_{\text {trans }}=3.0 \mathrm{~Hz}$ between $\mathrm{H} 1 \mathrm{a}$ and $\mathrm{H} 1$ and analogy with compound 6a-exo. $157 \mathrm{mg}$, (33\%) of another fraction containing a (1:3) mixture of isomers was isolated $\mathrm{Rf}: 0.15$ (Hexane/AcOEt, 6:1). Data for minor isomer are given from this mixture. Configuration was assigned as $6 \mathrm{c}$-endo $\left(1 \mathrm{R}^{\star}, 1 \mathrm{aS}^{\star}, 3 \mathrm{aS}^{\star}, 6 \mathrm{aS}^{\star}\right)$ based on: Coupling constant of $J_{\text {cis }}=9.3 \mathrm{~Hz}$ between $\mathrm{H} 1 \mathrm{a}$ and $\mathrm{H} 1$ and a strong NOE cross peak in the NOESY of these two protons as well as analogy with 6a-endo. 6c-exo: IR: 2978, 2939, $1724 \mathrm{~cm}^{-1} .{ }^{1} \mathrm{H}$ NMR $(400 \mathrm{MHz}$, $\left.\mathrm{CDCl}_{3}\right) \delta 4.23-4.08\left(\mathrm{~m}, 6 \mathrm{H}, \mathrm{CH}_{2} \mathrm{CH}_{3}\right), 2.72(\mathrm{~d}, \mathrm{~J}=15.1 \mathrm{~Hz}, 1 \mathrm{H}$, $\mathrm{CCHHC}), 2.68-2.58\left(\mathrm{~m}, 2 \mathrm{H}, \mathrm{CCHHCH}+\mathrm{CH}_{2} \mathrm{CHCH}_{2}\right), 2.63$ (d, J = 15.2 $\mathrm{Hz}, 1 \mathrm{H}, \mathrm{CCH} H \mathrm{C}), 2.30-2.19(\mathrm{~m}, 1 \mathrm{H}, \mathrm{CH} H \mathrm{C}=\mathrm{O}), 2.23(\mathrm{~d}, \mathrm{~J}=3.0 \mathrm{~Hz}, 1 \mathrm{H}$, $\left.\mathrm{CHCO}_{2}\right), 2.17(\mathrm{~d}, \mathrm{~J}=3.0 \mathrm{~Hz}, 1 \mathrm{H}, \mathrm{CHCHC}=\mathrm{O}), 2.15-2.05(\mathrm{~m}, 1 \mathrm{H}$, $\mathrm{CH} H \mathrm{C}=\mathrm{O}), 2.00-1.91(\mathrm{~m}, 1 \mathrm{H}, \mathrm{CCHHCH}), 1.24\left(\mathrm{~m}, 9 \mathrm{H}, \mathrm{CH}_{2} \mathrm{CH}_{3}\right) .{ }^{13} \mathrm{C}$ NMR (101 MHz, CDCl $)_{3} \delta 210.3\left(\mathrm{CH}_{2} \mathrm{C}=\mathrm{O}\right), 171.6\left(\mathrm{C}\left(\mathrm{CO}_{2} \mathrm{CH}_{2}\right)_{2}\right), 171.1$ $\left(\mathrm{C}\left(\mathrm{CO}_{2} \mathrm{CH}_{2}\right)_{2}\right), \quad 168.9 \quad\left(\mathrm{CHCO}_{2} \mathrm{CH}_{2}\right), \quad 61.8 \quad\left(\mathrm{C}\left(\mathrm{CO}_{2} \mathrm{CH}_{2}\right)_{2}\right), \quad 61.3$ $\left(\mathrm{CHCO}_{2} \mathrm{CH}_{2}\right), 59.6\left(\mathrm{C}\left(\mathrm{CO}_{2} \mathrm{CH}_{2}\right)_{2}\right), 46.0\left(\mathrm{CH}_{2} \mathrm{CCH}\right), 41.0\left(\mathrm{CHCO}_{2}\right), 40.8$ $\left(\mathrm{CCH}_{2} \mathrm{CH}\right), 40.0\left(\mathrm{CH}_{2} \mathrm{CHCH}_{2}\right), 39.7\left(\mathrm{CH}_{2} \mathrm{C}=\mathrm{O}\right), 34.6\left(\mathrm{CCH}_{2} \mathrm{C}\right), 30.0$
$(\mathrm{CHCHC}=\mathrm{O}), 14.2\left(\mathrm{CHCO}_{2} \mathrm{CH}_{2} \mathrm{CH}_{3}\right), 13.9\left(\mathrm{C}\left(\mathrm{CO}_{2} \mathrm{CH}_{2} \mathrm{CH}_{3}\right)_{2}\right)$. Anal. Calcd. for $\mathrm{C}_{18} \mathrm{H}_{24} \mathrm{O}_{7}$ (352.38 $\left.\mathrm{g} / \mathrm{mol}\right): \mathrm{C}, 61.4 ; \mathrm{H}, 6.9 \%$. Found: $\mathrm{C}, 61.2 ; \mathrm{H}, 6.7 \%$. 6c-endo: IR: 2978, 2939, $1724 \mathrm{~cm}^{-1}$. ${ }^{1} \mathrm{H}$ NMR (400 MHz, $\left.\mathrm{CDCl}_{3}\right) \delta 4.26-$ $4.07\left(\mathrm{~m}, 6 \mathrm{H}, \mathrm{CH}_{2} \mathrm{CH}_{3}\right), 2.83-2.70\left(\mathrm{~m}, 4 \mathrm{H}, \mathrm{CH}_{2} \mathrm{CHCH}_{2}+\mathrm{CCH} H \mathrm{CH}+\right.$ $\mathrm{CHHC}=\mathrm{O}+\mathrm{CHHC}), 2.43(\mathrm{~d}, \mathrm{~J}=9.3 \mathrm{~Hz}, 1 \mathrm{H}, \mathrm{CHCHC}=\mathrm{O}), 2.32-2.21(\mathrm{~m}$, $3 \mathrm{H}, \mathrm{CCHHCH}+\mathrm{CHHC}=\mathrm{O}+\mathrm{CHHC}), 2.13\left(\mathrm{~d}, \mathrm{~J}=9.1 \mathrm{~Hz}, 1 \mathrm{H}, \mathrm{CHCO}_{2}\right)$ $1.30-1.22\left(\mathrm{~m}, 9 \mathrm{H}, \mathrm{CH}_{2} \mathrm{CH}_{3}\right) .{ }^{13} \mathrm{C} \mathrm{NMR}\left(101 \mathrm{MHz}, \mathrm{CDCl}_{3}\right) \delta 212.0$ $\left(\mathrm{CH}_{2} \mathrm{C}=\mathrm{O}\right), \quad 171.1 \quad\left(\mathrm{CCO}_{2}\right), \quad 171.0 \quad\left(\mathrm{CCO}_{2}\right), \quad 169.6 \quad\left(\mathrm{CHCO}_{2}\right), \quad 61.9$ $\left(\mathrm{C}\left(\mathrm{CO}_{2} \mathrm{CH}_{2}\right)_{2}\right), 61.8\left(\mathrm{CHCO}_{2} \mathrm{CH}_{2}\right), 60.7\left(\mathrm{C}\left(\mathrm{CO}_{2} \mathrm{CH}_{2}\right)_{2}\right), 48.1\left(\mathrm{CH}_{2} \mathrm{CCH}\right)$, $47.3\left(\mathrm{CCH}_{2} \mathrm{C}\right.$ or $\mathrm{CCH}_{2} \mathrm{CH}$ or $\left.\mathrm{CHCH}_{2} \mathrm{C}=\mathrm{O}\right), 41.8\left(\mathrm{CCH}_{2} \mathrm{C}\right.$ or $\mathrm{CCH}_{2} \mathrm{CH}$ or $\left.\mathrm{CHCH}_{2} \mathrm{C}=\mathrm{O}\right), \quad 41.4 \quad(\mathrm{CHCHC}=\mathrm{O}), \quad 38.8 \quad\left(\mathrm{CCH}_{2} \mathrm{C}\right.$ or $\mathrm{CCH}_{2} \mathrm{CH}$ or $\left.\mathrm{CHCH}_{2} \mathrm{C}=\mathrm{O}\right), 36.7\left(\mathrm{CH}_{2} \mathrm{CHCH}_{2}\right), 32.2\left(\mathrm{CHCO}_{2}\right),\left(\mathrm{CHCO}_{2} \mathrm{CH}_{2} \mathrm{CH}_{3}\right), 14.0$ $\left(\mathrm{C}\left(\mathrm{CO}_{2} \mathrm{CH}_{2} \mathrm{CH}_{3}\right)_{2}\right)$.

1-tert-Butyl 5,5-diethyl 2-oxohexahydrocyclopropa [c]pentalene1,5,5(6H)-tricarboxylate, $6 \mathrm{~d}$ : From $350 \mathrm{mg}$ of diethyl $\mathbf{5 b}^{[12]}(1.31 \mathrm{mmol})$ and $408 \mathrm{mg}$ of salt $1 \mathrm{c}(1.44 \mathrm{mmol})$, a crude mixture of isomeric cyclopropanes was obtained (3:2). After chromatography (Hexane/AcOEt, 9:1), 179 mg (38\% yield) of a colorless oil (Rf: 0.13; Hexane/AcOEt, 9:1) were obtained. This isomer was assigned as $6 \mathrm{~d}$-exo (1S* $1 \mathrm{aS}^{\star}, 3 \mathrm{aS}^{\star}, 6 \mathrm{aS}^{\star}$ ) based on: Coupling constant of $J_{\text {trans }}=3.0 \mathrm{~Hz}$ between $\mathrm{H} 1 \mathrm{a}$ and $\mathrm{H} 1$. NOE cross peak in the NOESY of $\mathrm{H} 3 \mathrm{a}$ with $\mathrm{H} 1 \mathrm{a}$ and analogy with compound 6b-exo. $171 \mathrm{mg}$, (34\% yield) of another fraction containing a (1:3) mixture of isomers was isolated Rf: 0.11 (Hexane/AcOEt, 9:1). Data for minor isomer are given from this mixture Configuration was assigned as $6 \mathrm{~d}$-endo $\left(1 \mathrm{R}^{*}, 1 \mathrm{aS}^{\star}, 3 \mathrm{aS}^{\star}, 6 \mathrm{aS}^{\star}\right)$ based on: Coupling constant of $J_{\text {cis }}=9.2 \mathrm{~Hz}$ between $\mathrm{H} 1 \mathrm{a}$ and $\mathrm{H} 1$ and a strong NOE cross peak in the NOESY of these two protons. A cross peaks between $\mathrm{H} 1 \mathrm{a}$ with $\mathrm{H} 3 \mathrm{a}$ suggests this configuration. 6d-exo: IR: 2980, 2936, $1726 \mathrm{~cm}^{-1} .{ }^{1} \mathrm{H}$ NMR $\left(400 \mathrm{MHz}, \mathrm{CDCl}_{3}\right) \delta 4.28-4.15(\mathrm{~m}, 4 \mathrm{H}$, $\left.\mathrm{CH}_{2} \mathrm{CH}_{3}\right), 2.75$ (d, J = $\left.15.1 \mathrm{~Hz}, 1 \mathrm{H}, \mathrm{CHHC}\right), 2.70-2.59(\mathrm{~m}, 2 \mathrm{H}$, $\left.\mathrm{CCHHCH}+\mathrm{CH}_{2} \mathrm{CHCH}_{2}\right), 2.62(\mathrm{~d}, \mathrm{~J}=14.7 \mathrm{~Hz}, 1 \mathrm{H}, \mathrm{CHHC}), 2.28-2.19$ $(\mathrm{m}, 1 \mathrm{H}, \mathrm{CH} H \mathrm{C}=\mathrm{O}), 2.20\left(\mathrm{~d}, \mathrm{~J}=3.0 \mathrm{~Hz}, 1 \mathrm{H}, \mathrm{CHCO}_{2}\right), 2.14-2.07(\mathrm{~m}, 1 \mathrm{H}$, $\mathrm{CHHC}=\mathrm{O}), 2.11(\mathrm{~d}, \mathrm{~J}=3.0 \mathrm{~Hz}, 1 \mathrm{H}, \mathrm{CHCHC}=\mathrm{O}), 2.04-1.95(\mathrm{~m}, 1 \mathrm{H}$, $\mathrm{CCHHCH}), 1.46\left(\mathrm{~s}, 9 \mathrm{H}, \mathrm{C}\left(\mathrm{CH}_{3}\right)_{3}\right), 1.26\left(\mathrm{t}, \mathrm{J}=7.1 \mathrm{~Hz}, 3 \mathrm{H}, \mathrm{CH}_{2} \mathrm{CH}_{3}\right), 1.24$ $\left(\mathrm{t}, \mathrm{J}=7.1 \mathrm{~Hz}, 3 \mathrm{H}, \mathrm{CH}_{2} \mathrm{CH}_{3}\right) .{ }^{13} \mathrm{C} \mathrm{NMR}\left(101 \mathrm{MHz}, \mathrm{CDCl}_{3}\right) \delta 210.7$ $\left(\mathrm{CH}_{2} \mathrm{C}=\mathrm{O}\right), \quad 171.7\left(\mathrm{CO}_{2} \mathrm{CH}_{2}\right), \quad 171.2\left(\mathrm{CO}_{2} \mathrm{CH}_{2}\right), \quad 168.0\left(\mathrm{CO}_{2} \mathrm{C}\right), 82.0$

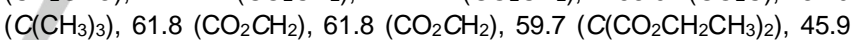
$\left(\mathrm{CH}_{2} \mathrm{C}(\mathrm{CH})_{2}\right), 41.0\left(\mathrm{CHCO}_{2}\right), 40.9\left(\mathrm{CCH}_{2} \mathrm{CH}\right), 40.0\left(\mathrm{CH}_{2} \mathrm{CHCH}_{2}\right), 39.8$ $\left(\mathrm{CH}_{2} \mathrm{C}=\mathrm{O}\right), 34.6\left(\mathrm{CCH}_{2} \mathrm{C}\right), 31.2(\mathrm{CHCHC}=\mathrm{O}), 28.1\left(\mathrm{C}_{\left.\left(\mathrm{CH}_{3}\right)_{3}\right),} 14.0\right.$ $\left(\mathrm{CH}_{2} \mathrm{CH}_{3}\right)$. Anal. Calcd. for $\mathrm{C}_{20} \mathrm{H}_{28} \mathrm{O}_{7}(380.43 \mathrm{~g} / \mathrm{mol}): \mathrm{C}, 63.1 ; \mathrm{H}, 7.4 \%$. Found: C, 62.9; H, $7.1 \%$. 6d-endo: IR: 2980, 2936, 1726. ${ }^{1} \mathrm{H}$ NMR (400 $\left.\mathrm{MHz}, \mathrm{CDCl}_{3}\right) \delta 4.23-4.15\left(\mathrm{~m}, 4 \mathrm{H}, \mathrm{CH}_{2} \mathrm{CH}_{3}\right), 2.78-2.58(\mathrm{~m}, 3 \mathrm{H}$, $\left.\mathrm{CCHHCH}+\mathrm{CH} H \mathrm{C}=\mathrm{O}+\mathrm{CH}_{2} \mathrm{CHCH}_{2}\right), 2.76(\mathrm{~d}, \mathrm{~J}=13.9 \mathrm{~Hz}, 1 \mathrm{H}, \mathrm{CH} H \mathrm{C})$, $2.37(\mathrm{~d}, \mathrm{~J}=9.2 \mathrm{~Hz}, 1 \mathrm{H}, \mathrm{CHCHC}=\mathrm{O}), 2.30-2.18(\mathrm{~m}, 2 \mathrm{H},+\mathrm{CCH} H \mathrm{HH}+$ $\mathrm{CH} H \mathrm{C}=\mathrm{O}), 2.28(\mathrm{~d}, \mathrm{~J}=13.7 \mathrm{~Hz}, 1 \mathrm{H}, \mathrm{CHHC}), 2.04(\mathrm{~d}, \mathrm{~J}=9.3 \mathrm{~Hz}, 1 \mathrm{H}$, $\left.\mathrm{CHCO}_{2}\right), 1.43\left(\mathrm{~s}, 9 \mathrm{H}, \mathrm{C}\left(\mathrm{CH}_{3}\right)_{3}\right), 1.26\left(\mathrm{t}, \mathrm{J}=7.1 \mathrm{~Hz}, 3 \mathrm{H}, \mathrm{CH}_{2} \mathrm{CH}_{3}\right), 1.24(\mathrm{t}$ $\left.\mathrm{J}=7.1 \mathrm{~Hz}, 3 \mathrm{H}, \mathrm{CH}_{2} \mathrm{CH}_{3}\right) .{ }^{13} \mathrm{C}$ NMR $\left(101 \mathrm{MHz}, \mathrm{CDCl}_{3}\right) \delta 212.0\left(\mathrm{CH}_{2} \mathrm{C}=\mathrm{O}\right)$, $\left.171.2\left(\mathrm{CO}_{2} \mathrm{CH}_{2}\right), 171.0\left(\mathrm{CO}_{2} \mathrm{CH}_{2}\right), 168.6\left(\mathrm{CO}_{2} \mathrm{C}\right), 82.0\left(\mathrm{C}_{\left(\mathrm{CH}_{3}\right)}\right)_{3}\right), 61.9$ $\left(\mathrm{CO}_{2} \mathrm{CH}_{2}\right), 61.7\left(\mathrm{CO}_{2} \mathrm{CH}_{2}\right), 60.8\left(\mathrm{C}\left(\mathrm{CO}_{2} \mathrm{CH}_{2} \mathrm{CH}_{3}\right)_{2}\right), 47.8\left(\mathrm{CH}_{2} \mathrm{C}(\mathrm{CH})_{2}\right)$, $47.3\left(\mathrm{CCH}_{2} \mathrm{CH}\right), 41.9\left(\mathrm{CH}_{2} \mathrm{C}=\mathrm{O}\right), 41.1\left(\mathrm{CHCO}_{2}\right), 38.9\left(\mathrm{CCH}_{2} \mathrm{C}\right), 36.5$ $\left(\mathrm{CH}_{2} \mathrm{CHCH}_{2}\right), 33.5(\mathrm{CHCHC}=\mathrm{O}), 28.0\left(\mathrm{C}\left(\mathrm{CH}_{3}\right)_{3}\right), 14.0\left(\mathrm{CH}_{2} \mathrm{CH}_{3}\right)$.

Ethyl 5-oxo-2-tosyloctahydrocyclopropa[1,5]cyclopenta[1,2c]pyrrole-6-carboxylate, 6e: From $500 \mathrm{mg}$ of $5 c^{[12]}(1.98 \mathrm{mmol})$ and 500 $\mathrm{mg}$ of salt $1 \mathrm{a}(2.18 \mathrm{mmol})$, a crude mixture of isomeric cyclopropanes was obtained (3:1). After chromatography (Hexane/AcOEt, 3:2), $431 \mathrm{mg}$ ( $60 \%$ yield) of a colorless oil (Rf: 0.27 ; Hexane/AcOEt, 3:2), was isolated containing a 7:2 mixture of two diasteromers. Major isomer was assigned as $6 \mathrm{e}$-exo $\left(3 \mathrm{aS}^{*}, 5 \mathrm{aS}^{*}, 6 \mathrm{~S}^{\star}, 6 \mathrm{aR}^{*}\right)$ based on: Coupling constant of $J_{\text {trans }}=$ $2.9 \mathrm{~Hz}$ between $\mathrm{H} 5 \mathrm{a}$ and $\mathrm{H} 6$, NOE cross peak in the NOESY between $\mathrm{H} 3 \mathrm{a}$ and $\mathrm{H} 5 \mathrm{a}$ and the absence of any cross peak between $\mathrm{H} 3 \mathrm{a}$ and $\mathrm{H} 6$. Minor isomer was assigned as $6 \mathrm{e}$-endo $\left(3 \mathrm{aS}^{\star}, 5 \mathrm{aS}^{*}, 6 \mathrm{R}^{\star}, 6 \mathrm{aR}^{\star}\right)$ based on: Coupling constant of $J_{\text {cis }}=12.4 \mathrm{~Hz}$ between H5a and H6.IR: 2951, 2932, 
2864, $1726 \mathrm{~cm}^{-1}$. Data given from the mixture. 6e-exo: ${ }^{1} \mathrm{H}$ NMR (400 $\left.\mathrm{MHz}, \mathrm{CDCl}_{3}\right) \delta 7.65\left(\mathrm{~d}, \mathrm{~J}=8.1 \mathrm{~Hz}, 2 \mathrm{H}, \mathrm{CHCSO}_{2}\right), 7.31(\mathrm{~d}, \mathrm{~J}=8.1 \mathrm{~Hz}, 2 \mathrm{H}$ $\mathrm{CH}_{3} \mathrm{CCH}$ ), 4.12 (q, $J=7.2 \mathrm{~Hz}, 2 \mathrm{H}, \mathrm{CO}_{2} \mathrm{CH}_{2} \mathrm{CH}_{3}$ ), 3.87 (dd, $J=9.7,7.4$ $\mathrm{Hz}, 1 \mathrm{H}, \mathrm{NCHHCH}$ ), 3.70 (d, J = $11.5 \mathrm{~Hz}, 1 \mathrm{H}, \mathrm{CH} H \mathrm{C}$, major), 3.46 (d, J = $11.5 \mathrm{~Hz}, 1 \mathrm{H}, \mathrm{CH} H \mathrm{C}), 2.82-2.73\left(\mathrm{~m}, 1 \mathrm{H}, \mathrm{CH}_{2} \mathrm{CHCH}_{2}\right), 2.70-2.62(\mathrm{~m}$, $1 \mathrm{H}, \mathrm{NCHHCH}), 2.41\left(\mathrm{~s}, 3 \mathrm{H}, \mathrm{CCH}_{3}\right), 2.21(\mathrm{~d}, \mathrm{~J}=3.0 \mathrm{~Hz}, 1 \mathrm{H}, \mathrm{CHCHC}=\mathrm{O})$, $2.21-2.06(\mathrm{~m}, 1 \mathrm{H}, \mathrm{CHHC}=\mathrm{O}), 2.03\left(\mathrm{~d}, \mathrm{~J}=3.1 \mathrm{~Hz}, 1 \mathrm{H}, \mathrm{CHCO}_{2}\right), 2.02-$ $1.91(\mathrm{~m}, 1 \mathrm{H}, \mathrm{CH} \mathrm{HC}=\mathrm{O}), 1.23\left(\mathrm{t}, \mathrm{J}=7.2 \mathrm{~Hz}, 3 \mathrm{H}, \mathrm{CH}_{2} \mathrm{CH}_{3}\right) .{ }^{13} \mathrm{C}$ NMR (101 $\mathrm{MHz}, \mathrm{CDCl}_{3}$, from diastereomeric mixture) $\delta 208.3\left(\mathrm{CH}_{2} \mathrm{C}=\mathrm{O}\right), 168.3$ $\left(\mathrm{CO}_{2} \mathrm{CH}_{2}\right), \quad 143.9\left(\mathrm{CCH}_{3}\right), \quad 132.8\left(\mathrm{CSO}_{2}\right), \quad 129.8\left(\mathrm{CH}_{3} \mathrm{CCH}\right), 127.4$ $\left(\mathrm{CHCSO}_{2}\right), 61.6\left(\mathrm{CO}_{2} \mathrm{CH}_{2} \mathrm{CH}_{3}\right), 53.6\left(\mathrm{NCH}_{2} \mathrm{CH}\right), 48.3\left(\mathrm{CH}_{2} \mathrm{C}\right), 44.2$ $\left(\mathrm{CH}_{2} \mathrm{C}\right), \quad 39.6\left(\mathrm{CHCO}_{2}\right), \quad 39.0\left(\mathrm{CH}_{2} \mathrm{CHCH}_{2}\right), \quad 37.0 \quad\left(\mathrm{CH}_{2} \mathrm{C}=\mathrm{O}\right), \quad 28.8$ $(\mathrm{CHCHC}=\mathrm{O}), 21.4\left(\mathrm{CCH}_{3}\right), 14.0\left(\mathrm{CH}_{2} \mathrm{CH}_{3}\right) .6 e$-endo: ${ }^{1} \mathrm{H} \mathrm{NMR}(400 \mathrm{MHz}$, $\left.\mathrm{CDCl}_{3}\right) \delta 7.65\left(\mathrm{~d}, \mathrm{~J}=8.1 \mathrm{~Hz}, 2 \mathrm{H}, \mathrm{CHCSO}_{2}\right), 7.31(\mathrm{~d}, \mathrm{~J}=8.1 \mathrm{~Hz}, 2 \mathrm{H}$, $\left.\mathrm{CH}_{3} \mathrm{CCH}\right), 4.10-4.03\left(\mathrm{~m}, 2 \mathrm{H}, \mathrm{CO}_{2} \mathrm{CH}_{2} \mathrm{CH}_{3}\right), 3.75(\mathrm{dd}, \mathrm{J}=10.0,8.3 \mathrm{~Hz}$, $1 \mathrm{H}, \mathrm{NCHHCH}), 3.58$ (d, J = $10.7 \mathrm{~Hz}, 1 \mathrm{H}, \mathrm{CHHC}), 3.17$ (d, $\mathrm{J}=10.7 \mathrm{~Hz}$, $1 \mathrm{H}, \mathrm{CHHC}), 2.91(\mathrm{dd}, \mathrm{J}=10.0,7.8 \mathrm{~Hz}, 1 \mathrm{H}, \mathrm{NCHHCH}), 2.82-2.73(\mathrm{~m}$ $\left.1 \mathrm{H}, \mathrm{CH}_{2} \mathrm{CHCH}_{2}\right), 2.70-2.62(\mathrm{~m}, 1 \mathrm{H}, \mathrm{CHHC}=\mathrm{O}), 2.41\left(\mathrm{~s}, 3 \mathrm{H}, \mathrm{CCH}_{3}\right)$, $2.22(\mathrm{~d}, \mathrm{~J}=12.4 \mathrm{~Hz}, 1 \mathrm{H}, \mathrm{CHCHC}=\mathrm{O}), 2.17\left(\mathrm{~d}, \mathrm{~J}=12.4 \mathrm{~Hz}, 1 \mathrm{H}, \mathrm{CHCO}_{2}\right.$ ), $2.02-1.91(\mathrm{~m}, 1 \mathrm{H}, \mathrm{CH} H \mathrm{C}=\mathrm{O}), 1.20\left(\mathrm{t}, \mathrm{J}=7.1 \mathrm{~Hz}, 3 \mathrm{H}, \mathrm{CH}_{2} \mathrm{CH}_{3}\right) .{ }^{13} \mathrm{C}$ NMR (101 MHz, $\mathrm{CDCl}_{3}$, from diastereomeric mixture) $\delta 209.7\left(\mathrm{CH}_{2} \mathrm{C}=\mathrm{O}\right)$, $168.7\left(\mathrm{CO}_{2} \mathrm{CH}_{2}\right), 144.1\left(\mathrm{CCH}_{3}\right), 132.2\left(\mathrm{CSO}_{2}\right), 129.8\left(\mathrm{CH}_{3} \mathrm{CCH}\right), 127.6$ $\left(\mathrm{CHCSO}_{2}\right), 61.5\left(\mathrm{CO}_{2} \mathrm{CH}_{2} \mathrm{CH}_{3}\right), 55.2\left(\mathrm{NCH}_{2} \mathrm{CH}\right), 51.1\left(\mathrm{CH}_{2} \mathrm{C}\right), 46.4$ $\left(\mathrm{CH}_{2} \mathrm{C}\right), \quad 44.7 \quad\left(\mathrm{CH}_{2} \mathrm{C}=\mathrm{O}\right), \quad 39.8 \quad\left(\mathrm{CHCO}_{2}\right), \quad 36.6 \quad\left(\mathrm{CH}_{2} \mathrm{CHCH}_{2}\right), \quad 30.7$ $(\mathrm{CHCHC}=\mathrm{O}), 21.4\left(\mathrm{CCH}_{3}\right), 14.0\left(\mathrm{CH}_{2} \mathrm{CH}_{3}\right)$.

tert-Butyl 5-oxo-2-tosyloctahydrocyclopropa[1,5]cyclopenta[1,2c]pyrrole-6-carboxylate, 6f: From $250 \mathrm{mg}$ of $5 \mathbf{c}^{[12]}(0.90 \mathrm{mmol})$ and 282 $\mathrm{mg}$ of salt 1c $(0.99 \mathrm{mmol})$, a crude mixture of isomeric cyclopropanes was obtained (2:1). After chromatography (Hexane/AcOEt, 3:2), $162 \mathrm{mg}$ (48\% yield) of a colorless oil (Rf: 0.30 ; Hexane/AcOEt, 3:2), was isolated containing a 2:1 mixture of two diasteromers. Major isomer was assigned as $6 f-e x o\left(3 a S^{*}, 5 \mathrm{aS}^{*}, 6 \mathrm{~S}^{*}, 6 \mathrm{aR}^{\star}\right)$ based on: Coupling constant of $J_{\text {trans }}=$ $2.3 \mathrm{~Hz}$ between $\mathrm{H} 5 \mathrm{a}$ and $\mathrm{H} 6$ and on the analogy with compound $\mathbf{6 b}$-exo. Minor isomer was assigned as $6 \mathrm{f}-$ endo $\left(3 \mathrm{aS}^{\star}, 5 \mathrm{aS}^{*}, 6 \mathrm{R}^{\star}, 6 \mathrm{R}^{*}\right)$ based on: Coupling constant of $\mathrm{J}_{\text {cis }}=9.4 \mathrm{~Hz}$ between $\mathrm{H} 5 \mathrm{a}$ and $\mathrm{H} 6$ and on the analogy with compound 6b-endo. 6f-exo: IR: 2954, 2932, 2867, 1721 $\mathrm{cm}^{-1} .{ }^{1} \mathrm{H}$ NMR $\left(400 \mathrm{MHz}, \mathrm{CDCl}_{3}\right) \delta 7.70\left(\mathrm{~d}, \mathrm{~J}=8.1 \mathrm{~Hz}, 2 \mathrm{H}, \mathrm{CHCSO}_{2}\right)$, $7.36\left(\mathrm{~d}, \mathrm{~J}=8.0 \mathrm{~Hz}, 2 \mathrm{H}, \mathrm{CH}_{3} \mathrm{CCH}\right), 3.92(\mathrm{dd}, \mathrm{J}=9.7,7.4 \mathrm{~Hz}, 1 \mathrm{H}, \mathrm{CH} H \mathrm{CH})$ $3.78(\mathrm{~d}, \mathrm{~J}=11.6 \mathrm{~Hz}, 1 \mathrm{H}, \mathrm{NCHHC}), 3.45(\mathrm{~d}, \mathrm{~J}=11.5 \mathrm{~Hz}, 1 \mathrm{H}, \mathrm{NCHHC})$, $2.87-2.80\left(\mathrm{~m}, 1 \mathrm{H}, \mathrm{CH}_{2} \mathrm{CHCH}_{2}\right), 2.62(\mathrm{t}, \mathrm{J}=10.0 \mathrm{~Hz}, 1 \mathrm{H}, \mathrm{NCHHCH})$, $2.46\left(\mathrm{~s}, 3 \mathrm{H}, \mathrm{CCH}_{3}\right), 2.17(\mathrm{~d}, \mathrm{~J}=2.3 \mathrm{~Hz}, 1 \mathrm{H} \mathrm{CHCHC}=0), 2.21-2.15(\mathrm{~m}$, $1 \mathrm{H}, \mathrm{CHHC}=\mathrm{O}), 2.02$ (d, J = 2.3 Hz, $\left.1 \mathrm{H}, \mathrm{CHCO}_{2}\right), 1.99(\mathrm{~d}, \mathrm{~J}=19.4 \mathrm{~Hz}, 1 \mathrm{H}$ $\mathrm{CHHC}=\mathrm{O}), 1.45\left(\mathrm{~s}, 9 \mathrm{H}, \mathrm{C}\left(\mathrm{CH}_{3}\right)_{3}\right) .{ }^{13} \mathrm{C} \mathrm{NMR}\left(101 \mathrm{MHz}, \mathrm{CDCl}_{3}\right) \delta 208.8$ $\left(\mathrm{CH}_{2} \mathrm{C}=\mathrm{O}\right), \quad 167.4\left(\mathrm{CO}_{2} \mathrm{CH}_{2}\right), \quad 144.1 \quad\left(\mathrm{CCH}_{3}\right), 132.7 \quad\left(\mathrm{CSO}_{2}\right), 129.9$ $\left(\mathrm{CHCCH}_{3}\right), 127.6\left(\mathrm{CHCSO}_{2}\right), 82.8\left(\mathrm{C}_{\left.\left(\mathrm{CH}_{3}\right)_{3}\right),} 53.7\left(\mathrm{NCH}_{2} \mathrm{CH}\right), 48.4\right.$ $\left(\mathrm{CH}_{2} \mathrm{C}\right), 44.2\left(\mathrm{CH}_{2} \mathrm{C}\right), 39.7\left(\mathrm{CHCO}_{2}\right), 39.1\left(\mathrm{CH}_{2} \mathrm{CHCH}_{2}\right), 37.1\left(\mathrm{CH}_{2} \mathrm{C}=\mathrm{O}\right)$,

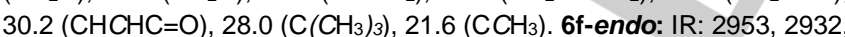
2865, $1725 \mathrm{~cm}^{-1} .{ }^{1} \mathrm{H}$ NMR (400 MHz, $\left.\mathrm{CDCl}_{3}\right) \delta 7.70(\mathrm{~d}, \mathrm{~J}=8.1 \mathrm{~Hz}, 2 \mathrm{H}$, $\mathrm{CHCSO}_{2}$ ), $7.36\left(\mathrm{~d}, \mathrm{~J}=8.0 \mathrm{~Hz}, 2 \mathrm{H}, \mathrm{CH}_{3} \mathrm{CCH}\right.$ ), 3.82 (dd, $\mathrm{J}=9.5,7.8 \mathrm{~Hz}$, $1 \mathrm{H}, \mathrm{CHHCH}), 3.65$ (d, J = $10.6 \mathrm{~Hz}, 1 \mathrm{H}, \mathrm{NCHHC}), 3.15(\mathrm{~d}, \mathrm{~J}=10.6 \mathrm{~Hz}$, $1 \mathrm{H}, \mathrm{NCH} H \mathrm{C}$ ), 2.91 (dd, $\mathrm{J}=9.5,8.0 \mathrm{~Hz}, 1 \mathrm{H}, \mathrm{NCH} H \mathrm{CH}), 2.87-2.80(\mathrm{~m}$, $1 \mathrm{H}, \mathrm{CH}_{2} \mathrm{CHCH}_{2}$ ), 2.68 (dd, J = 18.9, $7.7 \mathrm{~Hz}, 1 \mathrm{H}, \mathrm{CHHC}=\mathrm{O}$ ), 2.46 (s, 3H, $\left.\mathrm{CCH}_{3}\right), 2.36(\mathrm{~d}, \mathrm{~J}=9.4 \mathrm{~Hz}, 1 \mathrm{H}, \mathrm{CHCHC}=\mathrm{O}), 2.11-2.04(\mathrm{~m}, 1 \mathrm{H}$, $\mathrm{CHHC}=\mathrm{O}), 2.06\left(\mathrm{~d}, \mathrm{~J}=9.4 \mathrm{~Hz}, 1 \mathrm{H}, \mathrm{CHCO}_{2}\right), 1.42\left(\mathrm{~s}, 9 \mathrm{H}, \mathrm{C}\left(\mathrm{CH}_{3}\right)_{3}\right) .{ }^{13} \mathrm{C}$ NMR (101 MHz, CDCl $)_{3}$ ठ $210.0\left(\mathrm{CH}_{2} \mathrm{C}=\mathrm{O}\right), 167.8\left(\mathrm{CO}_{2} \mathrm{CH}_{2}\right), 144.2$ $\left(\mathrm{CCH}_{3}\right), \quad 132.3\left(\mathrm{CSO}_{2}\right), \quad 129.9\left(\mathrm{CH}_{3} \mathrm{CCH}\right), \quad 127.8\left(\mathrm{CHCSO}_{2}\right), \quad 82.7$ $\left(\mathrm{C}\left(\mathrm{CH}_{3}\right)_{3}\right), 55.4\left(\mathrm{NCH}_{2} \mathrm{CH}\right), 51.3\left(\mathrm{CH}_{2} \mathrm{C}\right), 46.3\left(\mathrm{CH}_{2} \mathrm{C}\right), 44.7\left(\mathrm{CH}_{2} \mathrm{C}=\mathrm{O}\right)$, $39.8\left(\mathrm{CHCO}_{2}\right), 36.7\left(\mathrm{CH}_{2} \mathrm{CHCH}_{2}\right), 32.1(\mathrm{CHCHC}=\mathrm{O}), 28.0\left(\mathrm{C}\left(\mathrm{CH}_{3}\right)_{3}\right)$, $21.6\left(\mathrm{CCH}_{3}\right)$.

\section{Acknowledgements}

Funding of this project by Spanish MINECO and Fondo Europeo de Desarrollo Regional (FEDER, grant No. CTQ2015-64624-R MINECO/FEDER) and FUSP-CEU (PC17/16) is acknowledged. A. L. thanks the Fundación San Pablo-CEU for pre-doctoral fellowship.

Keywords: sulfonium ylides • cyclopropanation • Microwave • pentalenene $\bullet$ stereoselective synthesis

[1] For reviews, see: a) X. L. Sun, Y. Tang, Acc. Chem. Res. 2008, 41, 937-948; b) E. M. McGarrigle, E. L. Myers, O. Illa, M. A. Shaw, S. L. Riches, V. K. Aggarwal, Chem. Rev. 2007, 107, 5841-5883; c) L.-X. Dai, X.-L. Hou, Y.-G. Zhou, Pure Appl. Chem. 1999, 71, 369-376; d) A.-H. Li, L.-X. Dai, V. K. Aggarwal, Chem. Rev. 1997, 97, 2341-2372.

[2] a) J.-F. Briere, P. Metzner in Organosulfur Chemistry in Asymmetric Synthesis, (Eds: T. Toru, C. Bolm), Wiley-VCH, Weinheim, 2008, pp 179-208; b) E. M. McGarrigle, V. K. Aggarwal, in Enantioselective Organocatalysis, (Ed: P. I. Dalko), Wiley-VCH, Weinheim, 2007, pp 357-389; c) V. K. Aggarwal, J. P. H. Charmant, D. Fuentes, J. N. Harvey, G. Hynd, D. Ohara, W. Picoul, R. Robiette, C. Smith, J.-L. Vasse, C. L. Winn, J. Am. Chem. Soc. 2006, 128, 2105-2114.

[3] a) R. Appel, N. Hartman, H. Mayr, J. Am. Chem. Soc. 2010, 132, 17894-17900; b) R. Appel, H. Mayr, Chem. Eur. J. 2010, 16, 8610-8614. a) S. L. Riches, C. Saha, N. F. Filgueira, E. Grange, E. M. McGarrigle, V. K. Aggarwal J. Am. Chem. Soc. 2010, 132, 7626-7630; b) D Janardanan, R. B. Sunoj, J. Org. Chem. 2007, 72, 331-341.

[5] V. K. Aggarwal, E. Grange, Chem. Eur. J. 2006, 12, 568-575

[6] a) P. Appukkattan, E. van der Eycken, Eur. J. Org. Chem. 2008 11331155 ; b) P. Nilsson, K. Olofsson, M. Larhed, Top. Curr. Chem. 2006, 266, 103-144; N. E. Leadbeater, Chem. Commun. 2005, 2881 2902.

[7] a) C. Jimeno, M. A. Pericas, H. P. Wessel, A. Alker, K. Mueller ChemMedChem 2011, 6, 1792-1795; b) F. Zhang, E. D. Moher, T. Y Zhang, Tetrahedron Lett. 2007, 48, 3277-3279; c) O. M. Rasmy, R. K. Vaid, M. J. Semo, E. C. Chelius, R. L. Robey, C. A. Alt, G. A. Rhodes, J. T. Vicenzi, Org. Process. Res. Dev. 2006, 10, 28-32; d) I. Collado, C. Domínguez, J. Ezquerra, C. Pedregal, J. A. Monn, Tetrahedron Lett. 1997, 38, 2133-2136.

[8] D. W. von Eggers, X. Zhao, J. Am. Chem. Soc. 2008, 130, 6430-6437.

[9] a) R. J. Goodridge, T. W. Hambley, R. K. Haynes, D. D. Ridley, J.Org Chem. 1988, 53, 2889-2898; b) R. Zhou, X. Deng, J. Zheng, Q. Shen X. Sun, Y. Tang, Chin. J. Chem. 2011, 29, 995-1000.

[10] The ee was measured by HPLC (Chiralpack AD-H, $4.6 \mathrm{~mm} \times 250 \mathrm{~mm}$ ) Optical rotation of the scalemic mixture was positive so the major enantiomer is assigned to $(1 S, 5 R, 6 S)$ following ref 5 .

[11] Reviews on triquinanes: a) G. Mehta, A. Srikrishna, Chem. Rev. 1997, 97, 671-720; b) L. A. Paquette, Top. Curr.Chem. 1984, 119, 1-158.

[12] Synthesis of 5a:D. H. Hua, J. Am. Chem. Soc. 1986, 108, 3837-3838. Synthesis of $\mathbf{5 b}$ and $\mathbf{5 c}$ : B. L. Pagenkopf, T. Livinghouse, J. Am. Chem. Soc. 1996, 118, 2285-2286.

[13] M. K. Pallerla, J. M. Fox, Org. Lett. 2007, 9, 5625-5628.

[14] Synthesis of salts $\mathbf{1 a - c}$ see ref 5 ; synthesis of $\mathbf{1 d}$, see ref. 3.

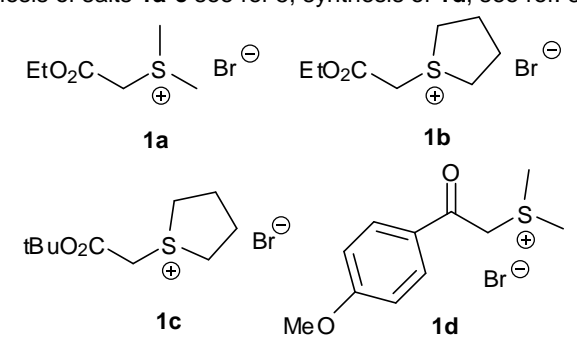


[15] K. Y. Lee, J. E. Na, J. Y. Lee, J. N. Kim, Bull. Korean Chem. Soc. 2004, $25,1280-1282$. 
Entry for the Table of Contents (Please choose one layout)

Layout 2:

\section{FULL PAPER}

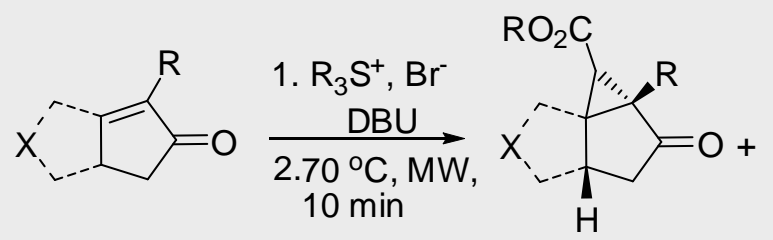

Ylide cyclopropanation reactions under MW mediation give excellent yields and selectivities after short reaction periods. Sterically hindered cyclopentenones and highly connected cyclopropa[c]pentalenes were synthesized in good yields, including a precursor of natural product pentalenene.
MW assisted ylide cyclopropanation*

Alberto López-Rodríguez, Gema Domínguez, Javier Pérez-Castells*

Page No. - Page No.

Microwave-Mediated Sulfonium Ylide Cyclopropanation. Stereoselective Synthesis of

Cyclopropa[c]pentalenes

*one or two words that highlight the emphasis of the paper or the field of the study 